\title{
Green Synthesis Characterization and Thermotropic Behaviour of O-Linked Glycopyranosides of Phenolic Esters
}

\author{
Saliha Nazir $\mathbb{D}^{1},{ }^{1}$ Dure Najaf Iqbal, ${ }^{2}$ Hina Daud, ${ }^{1}$ Ambreen Ghani $\mathbb{D}^{3},{ }^{3}$ Shagufta Nasir $\mathbb{D}^{1},{ }^{1}$ \\ Saima Naz, ${ }^{4}$ and Erum Akbar Hussain (iD ${ }^{1,5}$ \\ ${ }^{1}$ Department of Chemistry, Lahore College for Women University, Lahore 54000, Pakistan \\ ${ }^{2}$ Department of Chemistry, University of Lahore, Lahore 55150, Pakistan \\ ${ }^{3}$ Department of Chemistry, University of Education Lahore, Vehari Campus, Vehari 61100, Pakistan \\ ${ }^{4}$ Department of Chemistry, University of Education Lahore, Multan Campus, Multan, Pakistan \\ ${ }^{5}$ H.E.J Research Institute of Chemistry, University of Karachi, Karachi, Pakistan \\ Correspondence should be addressed to Saliha Nazir; nazirsaliha@gmail.com, Ambreen Ghani; ambreen.ghani@ \\ ue.edu.pk, and Erum Akbar Hussain; erum.hussain@gmail.com
}

Received 1 March 2020; Revised 12 June 2020; Accepted 13 June 2020; Published 31 July 2020

Academic Editor: Jolanta N. Latosinska

Copyright $\odot 2020$ Saliha Nazir et al. This is an open access article distributed under the Creative Commons Attribution License, which permits unrestricted use, distribution, and reproduction in any medium, provided the original work is properly cited.

Focusing on green chemistry protocols, a series of carbohydrate derivatives (5a-l) have been synthesized by Fischer glycosylation of $\alpha$-D-glucose, $\mathrm{D}$-xylose, and $\alpha$-maltose with several nonpolar phenolic ester aglycones (3a-d) derived from menthol by employing solid-supported $\mathrm{Si}-\mathrm{H}^{+}$as the catalyst. In order to study the extent of mesomorphism in target molecules, the thermotropic behaviour has been studied by using the thermoanalytic DSC/TGA technique and polarized optical microscope. Phase transitions in the DSC thermograms of 5a-l with two endothermic melting point peaks and various exothermic crystalline transitions exhibits the existence of mesophases. However, optical photomicrographs revealed that the new glycopyranosides formed smectic A phases. Moreover, all the compounds (3a-d and 5a-l) were confirmed by FTIR and ${ }^{1} \mathrm{H}$ NMR.

\section{Introduction}

Mesogens, the emerging scientific domain is thermodynamically stable, dimensionally ordered, flowing state of matter that exist between isotropic liquid and crystalline solid. They have been experiential for over hundred years but were first recognized systematically by Friedrich Reinitzer [1-3]. Glycosides are of special interest due to their role as thermotropic and lyotropic mesogens, surfactants, lubricants, solvents, drug delivery systems and membrane crystallizers in pharmaceutics, food, biotechnology and material chemistry [4]. The existence of mesogens primarily depends upon the nature of glycone used as a hydrophilic part, hydrophobic aglycone part attached to the polar carbohydrate, ether or the ester linkage and the effect of any rigid spacer or unsaturation thus imparting the mesophase behaviour to the overall molecule [5].

According to literature guidelines, glycosylation reactions have been executed by employing various solvents such as dichloromethane, tetrahydrofuran, toluene [6], dioxane $[7,8]$ and dimethylformamide $[9,10]$. The variety of catalyst ranges from the organic catalyst such as $\mathrm{N}$-iodosuccinimide, boron trifluoride etherate, 4-dimethylaminopyridine, pentamethyldiethylenetriamine, 1-ethyl-3-(3-dimethylaminopropyl) carbodiimide to the metal catalyst including tin tetrachloride and indium [11-20]. However, their toxicity, prohibitive prices and increased waste disposal costs add up to serious drawbacks for the large-scale applications. The environmental concerns addressed elimination of toxic solvents and reagents in the chemical reactions and doing them "neat" goes a long way towards achieving the goals of green atom economic chemistry [21,22].

Therefore, the aim of the present research deals with the solvent-free synthesis of twelve new glycosides by condensing $\alpha$-D-glucose, $\mathrm{D}$-xylose, and $\alpha$-maltose to phenolic esters as candidates possessing phase transitions and mesogenic behaviour. Preparation of the solid-supported Si$\mathrm{H}^{+}$catalyst and its characterization are imperative features 
of this work. First, direct condensation of mono-, di-, and trihydroxy phenolic acids with menthol was accomplished. Then, mesogenic nature of the molecule was achieved by chemical combination of hydrophobic esters and glycones by making some modifications in the reported glycosylation strategies.

\section{Experimental}

2.1. Materials and Methods. All reagents and solvents were of analytical grade with highest purity. L-Menthol, $\alpha$-Dglucose, D-xylose, and $\alpha$-maltose were purchased from Sigma-Aldrich and Merck. Melting points were determined with a Gallenkamp digital melting point apparatus with a maximum range of $360^{\circ} \mathrm{C}$. Reaction progress was observed by TLC on silica gel 60F-254 sheets (Merck) visualized with the mineral light UV lamp $(230 \mathrm{~V} \sim 50 / 60 \mathrm{~Hz})$. Silica gel mesh size 60-200 was used for catalyst preparation, while 230-400 mesh size was used for purification by column chromatography. Spectroanalytical techniques were used for the confirmation of chemical structures of all the products. FTIR spectra were scanned in a Shimadzu IR Tracer-100 FT-IR spectrometer. ${ }^{1} \mathrm{HNMR}$ spectra of all the compounds were recorded in ppm at $400 \mathrm{MHz}$ in $\mathrm{DMSO}-\mathrm{d}_{6}$ on the Bruker NMR spectrometer. The thermal behaviour and the phase transitions were analyzed by DSC/TGA, SDT-Q600TA Instrument, USA, from $25^{\circ} \mathrm{C}$ to $600^{\circ} \mathrm{C}$ at $10^{\circ} \mathrm{C} / \mathrm{min}$ heating rate (Exo Up). XRD patterns were recorded on D8 Discover diffractometer, Bruker, Germany. SEM micrographs were obtained using the ZEISS scanning electron microscope with the HDBSD detector. Optical textures were observed using the Polaroid Olympus camera-fitted optical microscope with a Mettler Toledo hot stage.

2.2. Preparation of Acid-Immobilized Silica Si-H $H^{+}$Catalyst. $\mathrm{Si}-\mathrm{H}^{+}$catalyst was prepared by stirring silica gel $(1 \mathrm{~g})$, mesh size 60-200, in $2 \mathrm{ml}$ dichloromethane for $15 \mathrm{~min}$ followed by addition of $0.75 \mathrm{ml}(1.39 \mathrm{~mol} \%)$ concentrated sulfuric acid (98\%) dropwise in silica and further stirred for an hour. The mixture was gradually dried at 60,100 , and then $120^{\circ} \mathrm{C}$ in an oven to get free-flowing $\mathrm{Si}-\mathrm{H}^{+}$catalyst.

2.3. Synthesis of Phenolic Ester Aglycones. Phenolic acids (1a-d) (1 mmol each), menthol (2) (0.468 g, $3 \mathrm{mmol})$ and Si$\mathrm{H}^{+}$catalyst $(0.294 \mathrm{~g}, 0.3$ mole $\%)$ were stirred at $80-100^{\circ} \mathrm{C}$ for 2.5 hours. TLC assisted to observe reaction progress using $30 \%$ ethyl acetate and n-hexane as eluent. After reaction completion, the contents were dissolved in ethyl acetate, filtered and dried to isolate the product. The catalyst was washed with dichloromethane and dried in vacuum. The crude product was then subjected to column chromatography and eluted with ethyl acetate and n-hexane as the mobile phase to afford pure $3 \mathrm{a}-\mathrm{d}$.

2.3.1. (1S,2R,5S)-2-Isopropyl-5-methylcyclohexyl 4-Hydroxybenzoate (3a). Yield: 89\%, pale yellow solid, mp: 145-148. FTIR: $\quad v_{\max }=3340.71, \quad 2954.95, \quad 1735.32,1446.61$,
$1165.00 \mathrm{~cm}^{-1} .{ }^{1} \mathrm{H}$ NMR $\left(400 \mathrm{MHz}, \mathrm{DMSO}-\mathrm{d}_{6}\right): \delta=0.80(\mathrm{~d}$, $J=7.2 \mathrm{~Hz}, 3 \mathrm{H}, \mathrm{H}-7), 0.85$ (d, $J=7.4 \mathrm{~Hz}, 3 \mathrm{H}, \mathrm{H}-9), 0.87$ (d, $J=7.5 \mathrm{~Hz}, 3 \mathrm{H}, \mathrm{H}-10), 1.35-1.40$ (m, $1 \mathrm{H}, \mathrm{H}-4), 1.43-1.48$ (m, $1 \mathrm{H}, \mathrm{H}-8), 1.50-1.54$ (m, 1H, H-1), $1.63-1.68$ (m, 2H, H-2), 1.70-1.75 (m, 2H, H-5), 1.77-1.79 (m, 2H, H-6), $3.44(\mathrm{dt}$, $J=11 \mathrm{~Hz}, 5 \mathrm{~Hz}, 1 \mathrm{H}, \mathrm{H}-3), 6.72\left(\mathrm{~d}, J=8.1 \mathrm{~Hz}, 2 \mathrm{H}, \mathrm{H}-1^{\prime}, \mathrm{H}-5^{\prime}\right)$, $7.88\left(\mathrm{~d}, J=8 \mathrm{~Hz}, 2 \mathrm{H}, \mathrm{H}-2^{\prime}, \mathrm{H}-4^{\prime}\right), 9.58$ (s, $\left.1 \mathrm{H}, \mathrm{OH}\right)$.

2.3.2. (1S,2R,5S)-2-Isopropyl-5-methylcyclohexyl 4-Hydroxy3-methoxybenzoate (3b). Yield: 93\%, white crystalline solid, mp: 130-135. FTIR: $\nu_{\max }=3371.57,2966.52,1731.27$, 1440.33, $1161.15 \mathrm{~cm}^{-1}$. ${ }^{1} \mathrm{H}$ NMR (400 MHz, DMSO-d $\left.\mathrm{d}_{6}\right)$ : $\delta=0.81(\mathrm{~d}, J=7.2 \mathrm{~Hz}, 3 \mathrm{H}, \mathrm{H}-7), 0.84(\mathrm{~d}, J=7.3 \mathrm{~Hz}, 3 \mathrm{H}, \mathrm{H}-9)$, 0.87 (d, $J=7.5 \mathrm{~Hz}, 3 \mathrm{H}, \mathrm{H}-10), 1.34-1.38$ (m, $1 \mathrm{H}, \mathrm{H}-4)$, 1.43-1.46 (m, 1H, H-8), 1.51-1.54 (m, 1H, H-1), 1.64-1.67 (m, 2H, H-2), 1.70-1.74 (m, 2H, H-5), 1.76-1.79 (m, 2H, H-6), 3.43 (dt, $J=11 \mathrm{~Hz}, 4.9 \mathrm{~Hz}, 1 \mathrm{H}, \mathrm{H}-3), 3.77$ (s, 3H, $\left.\mathrm{OCH}_{3}\right), 7.11\left(\mathrm{~d}, J=8 \mathrm{~Hz}, 1 \mathrm{H}, \mathrm{H}-4^{\prime}\right), 7.43(\mathrm{dd}, J=8 \mathrm{~Hz}, 3 \mathrm{~Hz}$, $\left.1 \mathrm{H}, \mathrm{H}-5^{\prime}\right), 7.54$ (d, $\left.J=2 \mathrm{~Hz}, 1 \mathrm{H}, \mathrm{H}-1^{\prime}\right), 9.50$ (s, 1H, OH).

2.3.3. (1S,2R,5S)-2-Isopropyl-5-methylcyclohexyl 4-Hydroxy3,5-dimethoxybenzoate (3c). Yield: 82\%, brown crystalline solid, mp: 108-110. FTIR: $\nu_{\max }=3336.85,2954.95,1734.05$, $1458.18,1118.71 \mathrm{~cm}^{-1}$. ${ }^{1} \mathrm{H}$ NMR $\left(400 \mathrm{MHz}, \mathrm{DMSO}-\mathrm{d}_{6}\right)$ : $\delta=0.79(\mathrm{~d}, J=7.2 \mathrm{~Hz}, 3 \mathrm{H}, \mathrm{H}-7), 0.83(\mathrm{~d}, J=7.4 \mathrm{~Hz}, 3 \mathrm{H}, \mathrm{H}-9)$, 0.87 (d, $J=7.4 \mathrm{~Hz}, 3 \mathrm{H}, \mathrm{H}-10), 1.33-1.37$ (m, $1 \mathrm{H}, \mathrm{H}-4)$, 1.43-1.45 (m, 1H, H-8), 1.52-1.57 (m, 1H, H-1), 1.62-1.65 (m, 2H, H-2), 1.71-1.74 (m, 2H, H-5), 1.76-1.78 (m, $2 \mathrm{H}$, H-6), 3.45 (dt, J=11 Hz, $5 \mathrm{~Hz}, 1 \mathrm{H}, \mathrm{H}-3), 3.70$ (s, 6H, $\left.\mathrm{O}-\mathrm{CH}_{3}\right), 6.92\left(\mathrm{~d}, J=2.5 \mathrm{~Hz}, 2 \mathrm{H}, \mathrm{H}-1^{\prime}, \mathrm{H}-5^{\prime}\right), 8.56(\mathrm{~s}, 1 \mathrm{H}$, $\mathrm{OH})$.

2.3.4. (1S,2R,5S)-2-Isopropyl-5-methylcyclohexyl (E)-3-(4Hydroxy-3-methoxyphenyl) Acrylate (3d). Yield: 96\%, pale yellow amorphous solid, mp: 245-250. FTIR: $v_{\max }=3610.74$, 2962.66, 671.23-956.69, 1728.93, 1427.32, 1180.44, 956.69, $671.23 \mathrm{~cm}^{-1} .{ }^{1} \mathrm{H}$ NMR $\left(400 \mathrm{MHz}, \mathrm{DMSO}-\mathrm{d}_{6}\right): \delta=0.82(\mathrm{~d}$, $J=7.2 \mathrm{~Hz}, 3 \mathrm{H}, \mathrm{H}-7), 0.84$ (d, $J=7.5 \mathrm{~Hz}, 3 \mathrm{H}, \mathrm{H}-9), 0.86$ (d, $J=7.4 \mathrm{~Hz}, 3 \mathrm{H}, \mathrm{H}-10), 1.34-1.35$ (m, $1 \mathrm{H}, \mathrm{H}-4), 1.43-1.47$ (m, $1 \mathrm{H}, \mathrm{H}-8), 1.53-1.57$ (m, 1H, H-1), 1.62-1.64 (m, 2H, H-2), 1.70-1.74 (m, 2H, H-5), 1.77-1.79 (m, 2H, H-6), 3.47 (dt, $\mathrm{J}=\mathrm{Hz}, \mathrm{Hz}, 1 \mathrm{H}, \mathrm{H}-3), 3.72\left(\mathrm{~s}, 3 \mathrm{H}, \mathrm{O}-\mathrm{CH}_{3}\right), 6.32$ (d, $\left.J=11.5 \mathrm{~Hz}, 1 \mathrm{H}, \mathrm{H}-8^{\prime}\right), 6.95\left(\mathrm{~d}, J=8 \mathrm{~Hz}, 1 \mathrm{H}, \mathrm{H}-4^{\prime}\right), 7.10$ (dd, $\left.J=8.3 \mathrm{~Hz}, 2.5 \mathrm{~Hz}, 1 \mathrm{H}, 5^{\prime}\right), 7.19\left(\mathrm{~d}, J=2 \mathrm{~Hz}, 1 \mathrm{H}, \mathrm{H}-1^{\prime}\right), 7.75$ (d, $\left.J=11.2 \mathrm{~Hz}, 1 \mathrm{H}, \mathrm{H}-7^{\prime}\right), 9.42(\mathrm{~s}, 1 \mathrm{H}, \mathrm{OH})$.

2.4. Synthesis of O-Glycopyranosides. Phenolic esters (3a-d) $(2 \mathrm{mmol})$ were treated with saccharides $(4 \mathrm{a}-\mathrm{c})(1 \mathrm{mmol})$ separately in the presence of $\mathrm{Si}-\mathrm{H}^{+}$catalyst $(0.098 \mathrm{~g}, 0.1$ mole $\%)$ at $80-100^{\circ} \mathrm{C}$. The reaction was monitored by TLC using dichloromethane and methanol $(5: 7)$ as eluent. Product spots were confirmed by spraying $2 \%$ ceric sulphate in $2 \mathrm{~N}$ $\mathrm{H}_{2} \mathrm{SO}_{4}$ followed by heating. The reaction contents were dissolved in methanol and filtered. After drying the filtrate, it was purified by column chromatography using gradient dichloromethane and methanol as the mobile phase to afford 
the amorphous products $(5 \mathrm{a}-\mathrm{d})$. The catalyst was separated as the residue, washed, and dried under vacuum.

2.4.1. (1S,2R,5S)-2-Isopropyl-5-methylcyclohexyl 4-(((2R, $3 R, 4 S, 5 S, 6 R)-3,4,5$-trihydroxy-6-(hydroxymethyl)tetrahydro 2H-Pyran-2-yl)oxy)benzoate (5a). Yield: $80 \%$, pale yellow. FTIR: $v_{\max }=3340.71,2927.94,1735.05,1562.34$, $1441.89,1158.42,540.37 \mathrm{~cm}^{-1}$. ${ }^{1} \mathrm{H}$ NMR $(400 \mathrm{MHz}$, DMSO$\left.\mathrm{d}_{6}\right): \delta=0.81(\mathrm{~d}, J=7.3 \mathrm{~Hz}, 3 \mathrm{H}, \mathrm{H}-7), 0.84(\mathrm{~d}, J=7.5 \mathrm{~Hz}, 3 \mathrm{H}$, $\mathrm{H}-9), 0.86$ (d, $J=7.5 \mathrm{~Hz}, 3 \mathrm{H}, \mathrm{H}-10), 1.34-1.37$ (m, $1 \mathrm{H}, \mathrm{H}-4)$, 1.41-1.47 (m, 1H, H-8), 1.52-1.56 (m, 1H, H-1), 1.63-1.67 (m, 2H, H-2), 1.71-1.74 (m, 2H, H-5), 1.76-1.79 (m, $2 \mathrm{H}$, H-6), 3.07 (dd, $\left.J=7.5 \mathrm{~Hz}, 3.4 \mathrm{~Hz}, 1 \mathrm{H}, \mathrm{H}-2^{\prime \prime}\right), 3.12-3.18$ (m, $\left.1 \mathrm{H}, \mathrm{H}-3^{\prime \prime}\right), 3.24-3.31\left(\mathrm{~m}, 1 \mathrm{H}, \mathrm{H}-4^{\prime \prime}\right), 3.45(\mathrm{dt}, J=11 \mathrm{~Hz}$, $4.5 \mathrm{~Hz}, 1 \mathrm{H}, \mathrm{H}-3), 3.48-3.50\left(\mathrm{~m}, 1 \mathrm{H}, \mathrm{H}-5^{\prime \prime}\right), 3.95(\mathrm{dd}$, $\left.J=14.5 \mathrm{~Hz}, 3 \mathrm{~Hz}, 2 \mathrm{H}, \mathrm{H}-6^{\prime \prime}\right), 4.15\left(\mathrm{~d}, J=7.4 \mathrm{~Hz}, 1 \mathrm{H}, \mathrm{H}-1^{\prime \prime}\right)$, $6.71\left(\mathrm{~d}, J=8.3 \mathrm{~Hz}, 2 \mathrm{H}, \mathrm{H}-1^{\prime}, \mathrm{H}-5^{\prime}\right), 7.88(\mathrm{~d}, J=8.5 \mathrm{~Hz}, 2 \mathrm{H}$, $\left.\mathrm{H}-2^{\prime}, \mathrm{H}-4^{\prime}\right), 9.55$ (s, 4H, OH).

2.4.2. (1S,2R,5S)-2-Isopropyl-5-methylcyclohexyl 4-(( $2 R$, $3 R, 4 S, 5 R)-3,4,5$-Trihydroxytetrahydro-2H-pyran-2-yl)oxy) benzoate (5b). Yield: $85 \%$, pale yellow. FTIR: $v_{\max }=3339.14,2920.23,1735.27,1564.63,1442.01,1147.85$, $555.50 \mathrm{~cm}^{-1} .{ }^{1} \mathrm{H}$ NMR $\left(400 \mathrm{MHz}\right.$, DMSO- $\left.\mathrm{d}_{6}\right): \delta=0.82(\mathrm{~d}$, $J=7.5 \mathrm{~Hz}, 3 \mathrm{H}, \mathrm{H}-7), 0.84(\mathrm{~d}, J=7.4 \mathrm{~Hz}, 3 \mathrm{H}, \mathrm{H}-9), 0.87(\mathrm{~d}$, $J=7.5 \mathrm{~Hz}, 3 \mathrm{H}, \mathrm{H}-10), 1.32-1.35$ (m, $1 \mathrm{H}, \mathrm{H}-4), 1.43-147$ (m, $1 \mathrm{H}, \mathrm{H}-8), 1.51-1.55$ (m, 1H, H-1), 1.61-1.64 (m, 2H, H-2), 1.72-1.73 (m, 2H, H-5), 1.75-1.80 (m, 2H, H-6), 3.10 (dd, $\left.J=7.5 \mathrm{~Hz}, 3.5 \mathrm{~Hz}, 1 \mathrm{H}, \mathrm{H}-2^{\prime \prime}\right), 3.17-3.20\left(\mathrm{~m}, 1 \mathrm{H}, \mathrm{H}-3^{\prime \prime}\right)$, $3.23-3.27\left(\mathrm{~m}, 1 \mathrm{H}, \mathrm{H}-4^{\prime \prime}\right), 3.34(\mathrm{dt}, J=11.5 \mathrm{~Hz}, 5 \mathrm{~Hz}, 1 \mathrm{H}$, $\mathrm{H}-3), 4.05-4.10\left(\mathrm{~m}, 2 \mathrm{H}, \mathrm{H}-5^{\prime \prime}\right), 4.20\left(\mathrm{~d}, J=7.5 \mathrm{~Hz}, 1 \mathrm{H}, \mathrm{H}-1^{\prime \prime}\right)$, $6.75\left(\mathrm{~d}, J=8 \mathrm{~Hz}, 2 \mathrm{H}, \mathrm{H}-1^{\prime}, \mathrm{H}-5^{\prime}\right), 7.89(\mathrm{~d}, J=8.5 \mathrm{~Hz}, 2 \mathrm{H}$, $\left.\mathrm{H}-2^{\prime}, \mathrm{H}-4^{\prime}\right), 9.49$ (s, 3H, OH).

2.4.3. (1S,2R,5S)-2-Isopropyl-5-methylcyclohexyl 4-(( $2 R$, $3 R, 4 R, 5 S, 6 R)-3,4-D i h y d r o x y-6-(h y d r o x y m e t h y l)-5-(((1 S, 2 R$, 3S,4R,5R)-2,3,4-trihydroxy-5-(hydroxymethyl)cyclohexyl) oxy)tetrahydro-2H-pyran-2-yl)oxy)benzoate (5c). Yield: $84 \%$, pale yellow gel-like. FTIR: $v_{\max }=3335.99,2927.94$, $1736.07,1562.03,1438.94,1147.06,544.08 \mathrm{~cm}^{-1} .{ }^{1} \mathrm{H}$ NMR $\left(400 \mathrm{MHz}\right.$, DMSO- $\left.\mathrm{d}_{6}\right): \delta=0.83(\mathrm{~d}, J=7.4 \mathrm{~Hz}, 3 \mathrm{H}, \mathrm{H}-7), 0.85$ (d, $J=7.5 \mathrm{~Hz}, 3 \mathrm{H}, \mathrm{H}-9), 0.88(\mathrm{~d}, J=7.2 \mathrm{~Hz}, 3 \mathrm{H}, \mathrm{H}-10)$, 1.30-1.35 (m, 1H, H-4), 1.41-1.44 (m, 1H, H-8), 1.52-1.54 (m, 1H, H-1), 1.62-1.65 (m, 2H, H-2), 1.73-1.77 (m, 2H, $\mathrm{H}-5), 1.79-1.82(\mathrm{~m}, 2 \mathrm{H}, \mathrm{H}-6), 3.06$ (dd, $J=7.6 \mathrm{~Hz}, 3.5 \mathrm{~Hz}$, $\left.2 \mathrm{H}, \mathrm{H}-2^{\prime \prime}\right), 3.12-3.17\left(\mathrm{~m}, 2 \mathrm{H}, \mathrm{H}-3^{\prime \prime}\right), 3.20-3.23(\mathrm{~m}, 2 \mathrm{H}$, $\left.\mathrm{H}-4^{\prime \prime}\right), 3.45(\mathrm{dt}, J=11.7 \mathrm{~Hz}, 5.5 \mathrm{~Hz}, 1 \mathrm{H}, \mathrm{H}-3), 4.18-4.20(\mathrm{~m}$, $\left.2 \mathrm{H}, \mathrm{H}-5^{\prime \prime}\right), 4.23\left(\mathrm{dd}, J=15 \mathrm{~Hz}, 3.2 \mathrm{~Hz}, 4 \mathrm{H}, \mathrm{H}-6^{\prime \prime}\right), 4.53(\mathrm{~d}$, $\left.J=7.6 \mathrm{~Hz}, 2 \mathrm{H}, \mathrm{H}-1^{\prime \prime}\right), 6.73\left(\mathrm{~d}, J=8.3 \mathrm{~Hz}, 2 \mathrm{H}, \mathrm{H}-1^{\prime}, \mathrm{H}-5^{\prime}\right)$, $7.86\left(\mathrm{~d}, J=7.6 \mathrm{~Hz}, 2 \mathrm{H}, \mathrm{H}-2^{\prime}, \mathrm{H}-4^{\prime}\right), 9.57$ (s, 7H, OH).

2.4.4. (1S,2R,5S)-2-Isopropyl-5-methylcyclohexyl 3-Methoxy4-(((2R,3R,4S,5S,6R)-3,4,5-trihydroxy-6-(hydroxymethyl)tetrahydro-2H-pyran-2-yl)oxy)benzoate (5d). Yield: $82 \%$, offwhite. FTIR: $v_{\max }=3309.85,2927.94,1730.22,1564.43$, $1420.60,1109.56,550.50 \mathrm{~cm}^{-1} .{ }^{1} \mathrm{H}$ NMR $(400 \mathrm{MHz}$, DMSO$\left.\mathrm{d}_{6}\right): \delta=0.81(\mathrm{~d}, J=7.5 \mathrm{~Hz}, 3 \mathrm{H}, \mathrm{H}-7), 0.85(\mathrm{~d}, J=7.6 \mathrm{~Hz}, 3 \mathrm{H}$,
H-9), 0.88 (d, $J=7.2 \mathrm{~Hz}, 3 \mathrm{H}, \mathrm{H}-10), 1.32-1.36(\mathrm{~m}, 1 \mathrm{H}, \mathrm{H}-4)$, 1.41-1.45 (m, 1H, H-8), 1.52-1.54 (m, 1H, H-1), 1.60-1.65 (m, 2H, H-2), 1.72-1.75 (m, 2H, H-5), 1.79-1.81 (m, 2H, H-6), 3.08 (dd, $J=7.5 \mathrm{~Hz}, 3.1 \mathrm{~Hz}, 1 \mathrm{H}, \mathrm{H}-2^{\prime \prime}$ ), $3.15-3.20$ (m, $\left.1 \mathrm{H}, \mathrm{H}-3^{\prime \prime}\right), 3.22-3.25\left(\mathrm{~m}, 1 \mathrm{H}, \mathrm{H}-4^{\prime \prime}\right), 3.47$ (dt, $J=10.6 \mathrm{~Hz}$, $5 \mathrm{~Hz}, 1 \mathrm{H}, \mathrm{H}-3), 3.77\left(\mathrm{~s}, 3 \mathrm{H}, \mathrm{OCH}_{3}\right), 4.06-4.11\left(\mathrm{~m}, 1 \mathrm{H}, \mathrm{H}-5^{\prime \prime}\right)$, $4.20\left(\mathrm{dd}, J=15.1 \mathrm{~Hz}, 2.8 \mathrm{~Hz}, 2 \mathrm{H}, \mathrm{H}-6^{\prime \prime}\right), 4.50(\mathrm{~d}, J=7.8 \mathrm{~Hz}$, $\left.1 \mathrm{H}, \mathrm{H}-1^{\prime \prime}\right), 7.11\left(\mathrm{~d}, J=8 \mathrm{~Hz}, 1 \mathrm{H}, \mathrm{H}-4^{\prime}\right), 7.43(\mathrm{dd}, J=8 \mathrm{~Hz}$, $\left.3 \mathrm{~Hz}, 1 \mathrm{H}, \mathrm{H}-5^{\prime}\right), 7.54$ (d, $\left.J=2 \mathrm{~Hz}, 1 \mathrm{H}, \mathrm{H}-1^{\prime}\right), 9.50(\mathrm{~s}, 1 \mathrm{H}$, $\mathrm{OH})$.

2.4.5. (1S,2 R,5S)-2-Isopropyl-5-methylcyclohexyl 3-Methoxy4-(((2R,3R,4S,5R)-3,4,5-trihydroxytetrahydro-2H-pyran-2-yl) oxy)benzoate (5e). Yield: 71\%, off-white. FTIR: $v_{\max }=3370.67,2966.50,1730.08,1560.35,1431.49,1109.14$, $583.03 \mathrm{~cm}^{-1} .{ }^{1} \mathrm{H}$ NMR $\left(400 \mathrm{MHz}\right.$, DMSO-d $\left.\mathrm{d}_{6}\right): \delta=0.83(\mathrm{~d}$, $J=7.5 \mathrm{~Hz}, 3 \mathrm{H}, \mathrm{H}-7), 0.85$ (d, $J=7.4 \mathrm{~Hz}, 3 \mathrm{H}, \mathrm{H}-9), 0.87$ (d, $J=7.5 \mathrm{~Hz}, 3 \mathrm{H}, \mathrm{H}-10), 1.31-1.34(\mathrm{~m}, 1 \mathrm{H}, \mathrm{H}-4), 1.42-1.47$ (m, $1 \mathrm{H}, \mathrm{H}-8), 1.50-1.53$ (m, 1H, H-1), 1.62-1.64 (m, 2H, H-2), 1.69-1.73 (m, 2H, H-5), 1.78-1.80 (m, 2H, H-6), 3.10 (dd, $\left.J=7.4 \mathrm{~Hz}, 3.5 \mathrm{~Hz}, 1 \mathrm{H}, \mathrm{H}-2^{\prime \prime}\right), 3.18-3.24\left(\mathrm{~m}, 1 \mathrm{H}, \mathrm{H}-3^{\prime \prime}\right)$, 3.26-3.37 (m, 1H, H-4"), $3.49(\mathrm{dt}, J=10.5 \mathrm{~Hz}, 4.9 \mathrm{~Hz}, 1 \mathrm{H}$, $\mathrm{H}-3), 3.60$ (s, 3H, $\left.\mathrm{OCH}_{3}\right), 4.20-4.24\left(\mathrm{~m}, 2 \mathrm{H}, \mathrm{H}-5^{\prime \prime}\right), 4.43$ (d, $\left.J=7.2 \mathrm{~Hz}, 1 \mathrm{H}, \mathrm{H}-1^{\prime \prime}\right), 7.15$ (d, $\left.J=8 \mathrm{~Hz}, 1 \mathrm{H}, \mathrm{H}-4^{\prime}\right), 7.45$ (dd, $\left.J=8.1 \mathrm{~Hz}, 3.5 \mathrm{~Hz}, 1 \mathrm{H}, \mathrm{H}-5^{\prime}\right), 7.51\left(\mathrm{~d}, J=2.1 \mathrm{~Hz}, 1 \mathrm{H}, \mathrm{H}-1^{\prime}\right)$, $9.34(\mathrm{~s}, 3 \mathrm{H}, \mathrm{OH})$.

2.4.6. (1S,2R,5S)-2-Isopropyl-5-methylcyclohexyl 4- $(((2 R, 3 R$, $4 R, 5 S, 6 R)-3,4-D i h y d r o x y-6$-(hydroxymethyl)-5-(((1S,2R,3S, 4R,5R)-2,3,4-trihydroxy-5-(hydroxymethyl)cyclohexyl)oxy) tetrahydro-2H-pyran-2-yl)oxy)-3-methoxybenzoate (5f). Yield: $80 \%$, off-white. FTIR: $v_{\max }=3378.09,2967.95$, $1729.87,1560.40,1430.97,1111.00,580.78 \mathrm{~cm}^{-1} .{ }^{1} \mathrm{H}$ NMR $\left(400 \mathrm{MHz}\right.$, DMSO- $\left.\mathrm{d}_{6}\right): \delta=0.82(\mathrm{~d}, J=7.4 \mathrm{~Hz}, 3 \mathrm{H}, \mathrm{H}-7), 0.85$ (d, $J=7.5 \mathrm{~Hz}, 3 \mathrm{H}, \mathrm{H}-9), 0.88(\mathrm{~d}, J=7.4 \mathrm{~Hz}, 3 \mathrm{H}, \mathrm{H}-10)$, 1.32-1.34 (m, 1H, H-4), 1.41-1.45 (m, 1H, H-8), 1.51-1.55 (m, 1H, H-1), 1.60-1.63 (m, 2H, H-2), 1.65-1.68 (m, 2H, $\mathrm{H}-5), 1.73-1.76(\mathrm{~m}, 2 \mathrm{H}, \mathrm{H}-6), 3.13(\mathrm{dd} J=7.5 \mathrm{~Hz}, 3.1 \mathrm{~Hz}, 2 \mathrm{H}$, $\left.\mathrm{H}-2^{\prime \prime}\right), 3.15-3.18$ (m, 2H, H-3"), 3.20-3.23 (m, 2H, H-4" $)$, $3.50(\mathrm{dt}, J=11.2 \mathrm{~Hz}, 5 \mathrm{~Hz}, 1 \mathrm{H}, \mathrm{H}-3), 3.63\left(\mathrm{~s}, 3 \mathrm{H}, \mathrm{OCH}_{3}\right)$, $4.21-4.23\left(\mathrm{~m}, 2 \mathrm{H}, \mathrm{H}-5^{\prime \prime}\right), 4.27(\mathrm{dd}, J=15.3 \mathrm{~Hz}, 3.5 \mathrm{~Hz}, 4 \mathrm{H}$, H-6"), 4.57 (d, $\left.J=7.4 \mathrm{~Hz}, 2 \mathrm{H}, \mathrm{H}-1^{\prime \prime}\right), 7.19$ (d, $J=8.2 \mathrm{~Hz}, 1 \mathrm{H}$, $\left.\mathrm{H}-4^{\prime}\right), 7.32\left(\mathrm{dd}, J=8 \mathrm{~Hz}, 4.5 \mathrm{~Hz}, 1 \mathrm{H}, \mathrm{H}-5^{\prime}\right), 7.53$ (d, $\left.J=2.5 \mathrm{~Hz}, 1 \mathrm{H}, \mathrm{H}-1^{\prime}\right), 9.61(\mathrm{~s}, 7 \mathrm{H}, \mathrm{OH})$.

2.4.7. (1S,2R,5S)-2-Isopropyl-5-methylcyclohexyl 3,5-Dimethoxy-4-(( $(2 R, 3 R, 4 S, 5 S, 6 R)-3,4,5$-trihydroxy-6-(hydroxy methyl)tetrahydro-2H-pyran-2-yl)oxy)benzoate (5g). Yield: $74 \%$, pale yellow, FTIR: $\nu_{\max }=3335.85,2957.35$, $1734.93,1562.30,1451.98,1118.41,559.36 \mathrm{~cm}^{-1} .{ }^{1} \mathrm{H}$ NMR $\left(400 \mathrm{MHz}\right.$, DMSO- $\left.\mathrm{d}_{6}\right): \delta=0.81(\mathrm{~d}, J=7.2 \mathrm{~Hz}, 3 \mathrm{H}, \mathrm{H}-7), 0.83$ (d, $J=7.5 \mathrm{~Hz}, 3 \mathrm{H}, \mathrm{H}-9), 0.85(\mathrm{~d}, J=7.4 \mathrm{~Hz}, 3 \mathrm{H}, \mathrm{H}-10)$, $1.30-1.34(\mathrm{~m}, 1 \mathrm{H}, \mathrm{H}-4), 1.44-1.47$ (m, $1 \mathrm{H}, \mathrm{H}-8), 1.53-1.57$ (m, $1 \mathrm{H}, \mathrm{H}-1), 1.63-1.67(\mathrm{~m}, 2 \mathrm{H}, \mathrm{H}-2), 1.71-1.75(\mathrm{~m}, 2 \mathrm{H}$, H-5), $1.76-1.79$ (m, 2H, H-6), 3.15 (dd, $J=7.4 \mathrm{~Hz}, 2.8 \mathrm{~Hz}$, $\left.1 \mathrm{H}, \mathrm{H}-2^{\prime \prime \prime}\right), 3.17-3.19\left(\mathrm{~m}, 1 \mathrm{H}, \mathrm{H}-3^{\prime \prime}\right), 3.21-3.25(\mathrm{~m}, 1 \mathrm{H}$, $\left.\mathrm{H}-4^{\prime \prime}\right), 3.52(\mathrm{dt}, J=12 \mathrm{~Hz}, 5 \mathrm{~Hz}, 1 \mathrm{H}, \mathrm{H}-3), 3.65(\mathrm{~s}, 6 \mathrm{H}$, 
O- $\left.\mathrm{CH}_{3}\right), 4.18-4.22\left(\mathrm{~m}, 1 \mathrm{H}, \mathrm{H}-5^{\prime \prime}\right), 4.25(\mathrm{dd}, J=15 \mathrm{~Hz}$, $\left.3.4 \mathrm{~Hz}, 2 \mathrm{H}, \mathrm{H}-6^{\prime \prime}\right), 4.60$ (d, J=8.1 Hz, $\left.1 \mathrm{H}, \mathrm{H}-1^{\prime \prime}\right), 7.20$ (d, $\left.J=2.5 \mathrm{~Hz}, 2 \mathrm{H}, \mathrm{H}-1^{\prime}, \mathrm{H}-5^{\prime}\right), 9.01(\mathrm{~s}, 4 \mathrm{H}, \mathrm{OH})$.

2.4.8. (1S,2R,5S)-2-Isopropyl-5-methylcyclohexyl 3,5-Dimethoxy-4-(( $2 R, 3 R, 4 S, 5 R)-3,4,5$-trihydroxytetrahydro-2H-pyran2-yl)oxy)benzoate (5h). Yield: 69\%, pale yellow. FTIR: $v_{\max }=3335.73,2954.95,1734.05,1543.44,1452.01,1099.71$, $559.41 \mathrm{~cm}^{-1}$. ${ }^{1} \mathrm{H}$ NMR $\left(400 \mathrm{MHz}, \mathrm{DMSO}_{-} \mathrm{d}_{6}\right): \delta=0.82(\mathrm{~d}$, $J=7.4 \mathrm{~Hz}, 3 \mathrm{H}, \mathrm{H}-7), 0.85$ (d, $J=7.6 \mathrm{~Hz}, 3 \mathrm{H}, \mathrm{H}-9), 0.87$ (d, $J=7.2 \mathrm{~Hz}, 3 \mathrm{H}, \mathrm{H}-10), 1.32-1.35(\mathrm{~m}, 1 \mathrm{H}, \mathrm{H}-4), 1.43-1.47$ (m, $1 \mathrm{H}, \mathrm{H}-8), 1.52-1.55$ (m, $1 \mathrm{H}, \mathrm{H}-1), 1.62-1.65$ (m, 2H, H-2), 1.72-1.74 (m, 2H, H-5), 1.76-1.79 (m, 2H, H-6), 3.18 (dd, $\left.J=7.6 \mathrm{~Hz}, 3.6 \mathrm{~Hz}, 1 \mathrm{H}, \mathrm{H}-2^{\prime \prime}\right), 3.20-3.24\left(\mathrm{~m}, 1 \mathrm{H}, \mathrm{H}-3^{\prime \prime}\right)$, $3.26-3.29\left(\mathrm{~m}, 1 \mathrm{H}, \mathrm{H}-4^{\prime \prime}\right), 3.49(\mathrm{dt}, J=11.6 \mathrm{~Hz}, 5 \mathrm{~Hz}, 1 \mathrm{H}$, $\mathrm{H}-3), 3.60$ (s, 6H, O-CH $\mathrm{CH}_{3}, 4.17-4.21$ (m, 2H, H-5 $\left.{ }^{\prime \prime}\right), 4.49$ (d, $\left.J=7.9 \mathrm{~Hz}, 1 \mathrm{H}, \mathrm{H}-1^{\prime \prime}\right), 6.69\left(\mathrm{~d}, J=2.7 \mathrm{~Hz}, 2 \mathrm{H}, \mathrm{H}-1^{\prime}, \mathrm{H}-5^{\prime}\right)$, $9.64(\mathrm{~s}, 3 \mathrm{H}, \mathrm{OH})$.

2.4.9. (1S,2R,5S)-2-Isopropyl-5-methylcyclohexyl 4-(( $2 R, 3 R$, $4 R, 5 S, 6 R)-3,4-D i h y d r o x y-6-(h y d r o x y m e t h y l)-5-(((1 S, 2 R, 3 S$, $4 R, 5 R)-2,3,4$-trihydroxy-5-(hydroxymethyl)cyclohexyl)oxy) tetrahydro-2H-pyran-2-yl)oxy)-3,5-dimethoxybenzoate (5i). Yield: 68\%, gel-like. FTIR: $\nu_{\max }=3336.05,2954.02,1735.01$, 1548.08, 1451.76, 1120.71, 560.02-cm ${ }^{-1} .{ }^{1} \mathrm{H}$ NMR $(400 \mathrm{MHz}$, DMSO- $\left.\mathrm{d}_{6}\right): \delta=0.80(\mathrm{~d}, J=7.5 \mathrm{~Hz}, 3 \mathrm{H}, \mathrm{H}-7), 0.83(\mathrm{~d}$, $J=7.4 \mathrm{~Hz}, 3 \mathrm{H}, \mathrm{H}-9), 0.85$ (d, $J=7.4 \mathrm{~Hz}, 3 \mathrm{H}, \mathrm{H}-10), 1.30-1.34$ (m, 1H, H-4), 1.41-1.45 (m, 1H, H-8), 1.50-1.54 (m, 1H, $\mathrm{H}-1), 1.60-1.64(\mathrm{~m}, 2 \mathrm{H}, \mathrm{H}-2), 1.71-1.73(\mathrm{~m}, 2 \mathrm{H}, \mathrm{H}-5)$, 1.75-1.78 (m, 2H, H-6), $3.16(\mathrm{dd}, J=7.4 \mathrm{~Hz}, 3.5 \mathrm{~Hz}, 2 \mathrm{H}$, $\left.\mathrm{H}-2^{\prime \prime}\right)$, 3.18-3.20 (m, 2H, H-3"), 3.26-3.29 (m, 2H, H-4" ), 3.47 (dt, $J=11.5 \mathrm{~Hz}, 4.5 \mathrm{~Hz}, 1 \mathrm{H}, \mathrm{H}-3), 3.73$ (s, 6H, O-CH ${ }_{3}$, $4.14-4.21\left(\mathrm{~m}, 2 \mathrm{H}, \mathrm{H}-5^{\prime \prime}\right), 4.23(\mathrm{dd}, J=15.3 \mathrm{~Hz}, 4.3 \mathrm{~Hz}, 4 \mathrm{H}$, H-6 $\left.{ }^{\prime \prime}\right), 4.48$ (d, J=8 Hz, $\left.2 \mathrm{H}, \mathrm{H}-1^{\prime \prime}\right), 6.71(\mathrm{~d}, J=2.5 \mathrm{~Hz}, 2 \mathrm{H}$, $\left.\mathrm{H}-1^{\prime}, \mathrm{H}-5^{\prime}\right), 9.51$ (s, 7H, OH).

2.4.10. (1S,2R,5S)-2-Isopropyl-5-methylcyclohexyl (E)-3-(3Methoxy-4-(((2R,3R,4S,5S,6R)-3,4,5-trihydroxy-6-(hydroxymethyl)tetrahydro-2H-pyran-2-yl)oxy)phenyl)acrylate (5j). Yield: $83 \%$, pale yellow. FTIR: $\nu_{\max }=3610.71,2962.66$, $1728.75, \quad 1540.45, \quad 1426.76,1181.40, \quad 906.69,660.73$, $540.57 \mathrm{~cm}^{-1}$. ${ }^{1} \mathrm{H}$ NMR $\left(400 \mathrm{MHz}, \mathrm{DMSO}_{-} \mathrm{d}_{6}\right): \delta=0.79(\mathrm{~d}$, $J=7.4 \mathrm{~Hz}, 3 \mathrm{H}, \mathrm{H}-7), 0.82$ (d, $J=7.5 \mathrm{~Hz}, 3 \mathrm{H}, \mathrm{H}-9), 0.86$ (d, $J=7.6 \mathrm{~Hz}, 3 \mathrm{H}, \mathrm{H}-10), 1.33-1.37$ (m, $1 \mathrm{H}, \mathrm{H}-4), 1.41-1.45$ (m, $1 \mathrm{H}, \mathrm{H}-8), 1.50-1.56$ (m, 1H, H-1), $1.62-1.65$ (m, 2H, H-2), 1.73-1.75 (m, 2H, H-5), 1.76-1.78 (m, 2H, H-6), 3.19 (dd, $\left.J=7.3 \mathrm{~Hz}, 3.1 \mathrm{~Hz}, 1 \mathrm{H}, \mathrm{H}-2^{\prime \prime}\right), 3.20-3.23\left(\mathrm{~m}, 1 \mathrm{H}, \mathrm{H}-3^{\prime \prime}\right)$, 3.25-3.29 (m, $\left.1 \mathrm{H}, \mathrm{H}-4^{\prime \prime}\right), 3.44(\mathrm{dt}, J=11 \mathrm{~Hz}, 5.5 \mathrm{~Hz}, 1 \mathrm{H}$, $\mathrm{H}-3), 3.65$ (s, 3H, O-CH ${ }_{3}$ ), 4.22-4.24 (m, $\left.1 \mathrm{H}, \mathrm{H}-5^{\prime \prime}\right), 4.26$ $\left(\mathrm{dd}, J=15.1 \mathrm{~Hz}, 3.5 \mathrm{~Hz}, 2 \mathrm{H}, \mathrm{H}-6^{\prime \prime}\right), 4.56(\mathrm{~d}, J=7.2 \mathrm{~Hz}, 1 \mathrm{H}$, $\left.\mathrm{H}^{\prime \prime} 1^{\prime \prime}\right), 6.32\left(\mathrm{~d}, J=11.5 \mathrm{~Hz}, 1 \mathrm{H}, \mathrm{H}-8^{\prime}\right), 6.97(\mathrm{~d}, J=7.8 \mathrm{~Hz}, 1 \mathrm{H}$, $\left.\mathrm{H}-4^{\prime}\right), 7.15$ (dd, $\left.J=8.5 \mathrm{~Hz}, 2.5 \mathrm{~Hz}, 1 \mathrm{H}, 5^{\prime}\right), 7.29$ (d, $J=2.5 \mathrm{~Hz}$, $1 \mathrm{H}, \mathrm{H}-1^{\prime}$ ), 7.60 (d, J=11.5 Hz, $\left.1 \mathrm{H}, \mathrm{H}-7^{\prime}\right), 9.48$ (s, $\left.4 \mathrm{H}, \mathrm{OH}\right)$.

2.4.11. (1S,2R,5S)-2-Isopropyl-5-methylcyclohexyl (E)-3-(3Methoxy-4-(((2R,3R,4S,5R)-3,4,5-trihydroxytetrahydro- $2 \mathrm{H}$ pyran-2-yl)oxy)phenyl)acrylate (5k). Yield: 76\%, pale yellow. FTIR: $v_{\max }=3611.04,2960.40,1728.93,1543.50$, $1427.32,1176.44,960.05,676.23,533.20-\mathrm{cm}^{-1} .{ }^{1} \mathrm{H}$ NMR $\left(400 \mathrm{MHz}, \mathrm{DMSO}-\mathrm{d}_{6}\right): \delta=0.82(\mathrm{~d}, J=7.3 \mathrm{~Hz}, 3 \mathrm{H}, \mathrm{H}-7), 0.84$ (d, J=7.2 Hz, 3H, H-9), 0.87 (d, J=7.6 Hz, 3H, H-10), $1.32-1.35$ (m, 1H, H-4), 1.42-1.47 (m, 1H, H-8), 1.52-1.57 (m, 1H, H-1), 1.60-1.64 (m, 2H, H-2), 1.71.1.74 (m, 2H, H-5), 1.76-1.80 (m, 2H, H-6), 3.17 (dd, J=7.2 Hz, $3.4 \mathrm{~Hz}$, $\left.1 \mathrm{H}, \mathrm{H}-2^{\prime \prime}\right), 3.20-3.24\left(\mathrm{~m}, 1 \mathrm{H}, \mathrm{H}-3^{\prime \prime}\right), 3.26-3.30(\mathrm{~m}, 1 \mathrm{H}$, $\left.\mathrm{H}-4^{\prime \prime}\right), 3.45$ (dt, $\left.J=11.5 \mathrm{~Hz}, 5.5 \mathrm{~Hz}, 1 \mathrm{H}, \mathrm{H}-3\right), 3.57$ (s, $3 \mathrm{H}$, $\left.\mathrm{O}-\mathrm{CH}_{3}\right), 4.25-4.27\left(\mathrm{~m}, 2 \mathrm{H}, \mathrm{H}-5^{\prime \prime}\right), 4.51(\mathrm{~d}, J=7.4 \mathrm{~Hz}, 1 \mathrm{H}$, H-1"), 6.29 (d, $\left.J=11 \mathrm{~Hz}, 1 \mathrm{H}, \mathrm{H}-8^{\prime}\right), 6.95(\mathrm{~d}, J=8 \mathrm{~Hz}, 1 \mathrm{H}$, $\left.\mathrm{H}-4^{\prime}\right), 7.20\left(\mathrm{dd}, J=8 \mathrm{~Hz}, 3.1 \mathrm{~Hz}, 1 \mathrm{H}, 5^{\prime}\right), 7.33(\mathrm{~d}, J=2.3 \mathrm{~Hz}$, $1 \mathrm{H}, \mathrm{H}-1^{\prime}$ ), 7.57 (d, J=11.5 Hz, 1H, H-7'), 9.77 (s, 3H, OH).

2.4.12. (1S,2R,5S)-2-Isopropyl-5-methylcyclohexyl (E)-3-(4$(((2 R, 3 R, 4 R, 5 S, 6 R)-3,4-D i h y d r o x y-6-(h y d r o x y m e t h y l)-5-(((1 S$, $2 R, 3 S, 4 R, 5 R)-2,3,4$-trihydroxy-5-(hydroxymethyl)cyclohexyl) oxy)tetrahydro-2H-pyran-2-yl)oxy)-3-methoxyphenyl)acrylate (5l). Yield: 65\%, yellow gel-like. FTIR: $v_{\max }=3610.74$, 2962.68, 1729.01, 1540.34, 1425.62, 1180.32, 956.79, 665.90,

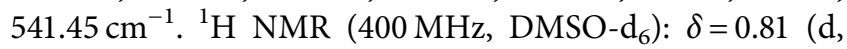
$J=7.5 \mathrm{~Hz}, 3 \mathrm{H}, \mathrm{H}-7), 0.83$ (d, $J=7.3 \mathrm{~Hz}, 3 \mathrm{H}, \mathrm{H}-9), 0.87$ (d, $J=7.5 \mathrm{~Hz}, 3 \mathrm{H}, \mathrm{H}-10), 1.30-1.34$ (m, $1 \mathrm{H}, \mathrm{H}-4), 1.41-1.45$ (m, $1 \mathrm{H}, \mathrm{H}-8), 1.53-1.55$ (m, 1H, H-1), $1.62-1.66$ (m, 2H, H-2), 1.72-1.75 (m, 2H, H-5), 1.79-1.82 (m, 2H, H-6), 3.12 (dd, $\left.J=7.4 \mathrm{~Hz}, \quad 3.6 \mathrm{~Hz}, \quad 2 \mathrm{H}, \quad \mathrm{H}-2^{\prime \prime}\right), \quad 3.24-3.27 \quad(\mathrm{~m}, \quad 2 \mathrm{H}$, H-3"),3.29-3.31 (m, 2H, H-4" $), 3.49(\mathrm{dt}, J=11 \mathrm{~Hz}, 4.5 \mathrm{~Hz}$, 1H, H-3), 3.61 (s, 3H, O- $\mathrm{CH}_{3}$ ), 4.15-4.20 (m, 2H, H-5"), 4.24 $\left(\mathrm{dd}, J=15.2 \mathrm{~Hz}, 4.1 \mathrm{~Hz}, 4 \mathrm{H}, \mathrm{H}-6^{\prime \prime}\right), 4.49$ (d, $J=8.1 \mathrm{~Hz}, 2 \mathrm{H}$, $\left.\mathrm{H}-1^{\prime \prime}\right), 6.25$ (d, $\left.J=10.6 \mathrm{~Hz}, 1 \mathrm{H}, \mathrm{H}-8^{\prime}\right), 6.98$ (d, $J=8 \mathrm{~Hz}, 1 \mathrm{H}$, $\left.\mathrm{H}-4^{\prime}\right), 7.14\left(\mathrm{dd}, J=8.1 \mathrm{~Hz}, 2 \mathrm{~Hz}, 1 \mathrm{H}, 5^{\prime}\right), 7.31$ (d, $J=2.5 \mathrm{~Hz}$, $\left.1 \mathrm{H}, \mathrm{H}-1^{\prime}\right), 7.49$ (d, J=11 Hz, 1H, H-7'), 9.48 (s, 7H, OH).

\section{Results and Discussion}

3.1. Selection of the Catalyst and Synthesis. The mesoporous solid-supported catalysts with large surface area and greater pore volume have high organic functionality on their surface and have been employed in a variety of organic reactions as environmentally safe and less-damaging green substitutes to conventional reagents [23]. Therefore, to promote environmental friendly processes, solid-supported catalyst has been employed for Fischer esterification followed by glycosylation to carry out direct condensation. In the first step, the hydroxyl group of menthol (2) was condensed with the carboxylic group of phenolic acids (1a-d) to yield nonpolar phenolic ester aglycones $(3 \mathrm{a}-\mathrm{d})$. Later, $3 \mathrm{a}-\mathrm{d}$ were glycosylated with polar glycones $(4 a-c)$ at the anomeric carbon to yield glycosides (5a-1) in appreciable yields (Figure 1). To make quantitative discussion of the effect, the role of different catalysts (Table 1) was explored using menthol (2) and 4-hydroxybenzoic acid (1a) as model substrates to synthesize $3 a$. Reaction parameters such as ratio of silica and acid as the effective catalyst, amount of the catalyst, temperature and reactant ratio were optimized under solvent-free conditions.

Several acids such as sulfuric acid $\left(\mathrm{H}_{2} \mathrm{SO}_{4}\right)$, sulfamic acid (SA), and $p$-toluenesulfonic acid (PTSA) were directly 
<smiles>[R]c1cc([R])c(O)c([18O])c1</smiles>

(1a-d)

(2)

$(3 a-c)$

(3d)

$$
\begin{aligned}
& \text { 1a } \mathrm{R}_{1}=-\mathrm{COOH} \\
& \mathrm{R}_{2}=\mathrm{R}_{3}=\mathrm{H} \\
& \text { lb } \mathrm{R}_{1}=-\mathrm{COOH} \\
& \mathrm{R}_{2}=-\mathrm{OCH}_{3} \\
& \mathrm{R}_{3}=\mathrm{H} \\
& \text { 1c } \mathrm{R}_{1}=-\mathrm{COOH} \\
& \mathrm{R}_{2}=\mathrm{R}_{3}=-\mathrm{OCH}_{3} \\
& \text { 1d } \mathrm{R}_{1}=\leadsto \mathrm{COOH} \\
& \mathrm{R}_{2}=\mathrm{H} \\
& \mathrm{R}_{3}=-\mathrm{OCH}_{3}
\end{aligned}
$$

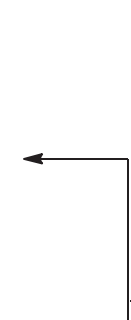

(5a-i)

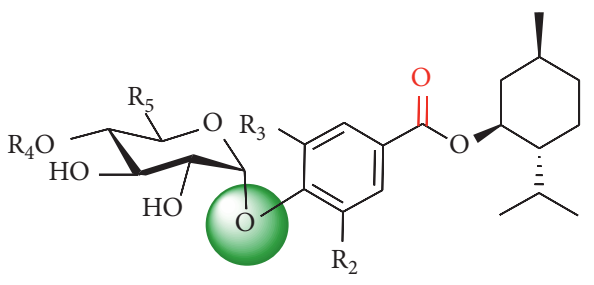

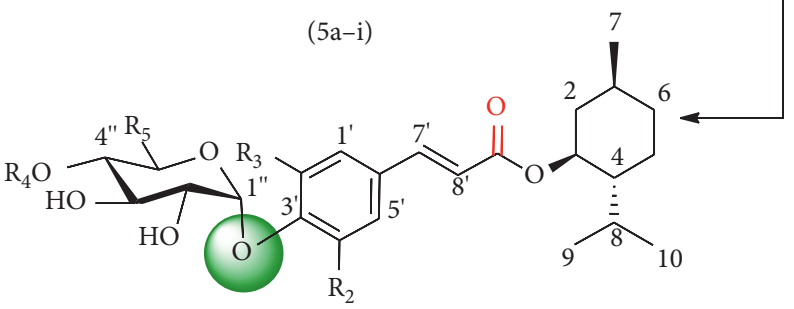

\begin{tabular}{|c|c|c|c|c|}
\hline Entry & Catalyst & Physical state & Time (hr) & Yield \% (3a) \\
\hline 1 & Conc. sulfuric acid $\left(\mathrm{H}_{2} \mathrm{SO}_{4}\right)$ & Viscous liquid & 1.5 & 5 \\
\hline 2 & Paratoluenesulfonic acid (PTSA) & White solid & 5.5 & 63 \\
\hline 3 & Sulfamic acid (SA) & White crystals & 4.5 & 67 \\
\hline 4 & Bentonite- $\mathrm{H}_{2} \mathrm{SO}_{4}$ & Grey solid & 2.5 & 71 \\
\hline 5 & Bentonite-PTSA & Grey solid & 5.5 & 55 \\
\hline 6 & Bentonite-SA & Grey solid & 5.5 & 60 \\
\hline 7 & Silica- $\mathrm{H}_{2} \mathrm{SO}_{4}\left(\mathrm{Si}-\mathrm{H}^{+}\right)$ & Grey powder & 2.5 & 86 \\
\hline 8 & Silica-PTSA & Grey powder & 5.5 & 56 \\
\hline 9 & Silica-SA & White powder & 4.5 & 54 \\
\hline
\end{tabular}

(ii)

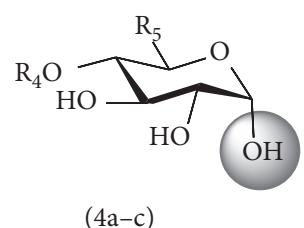

4a $\mathrm{R}_{4}=\mathrm{H}, \mathrm{R}_{5}=\mathrm{CH}_{2} \mathrm{OH}$

4b $\mathrm{R}_{4}=\mathrm{H}, \mathrm{R}_{5}=\mathrm{H}$

$4 \mathrm{c} \quad \mathrm{R}_{4}={ }_{\mathrm{HO}}^{\mathrm{HO}}$

(5j-1)

Figure 1: Synthesis of O-glycopyranosides. Reagents and conditions: (i) 0.3 mole $\%$ Si- $\mathrm{H}^{+}$catalyst, 1 mmole of 1a-d, 3 mmole of 2, 3-5 hours of stirring, and temp $80-100^{\circ} \mathrm{C}$; (ii) $0.1 \mathrm{~mole} \% \mathrm{Si}-\mathrm{H}^{+}$catalyst, 2 mmole of $3 \mathrm{a}-\mathrm{d}, 1$ mmole of $4 \mathrm{a}-\mathrm{c}, 3-7$ hours of stirring, and temp $80-100^{\circ} \mathrm{C}$.

TABLE 1: Optimization of the catalyst for the preparation of phenolic ester 3a using the direct condensation reaction.

Reagents and conditions: 4-hydroxybenzoic acid ( 1 mmole), menthol ( 3 mmole), 0.3 mole \% catalyst, and temp $80^{\circ} \mathrm{C}$.

employed for Fischer esterification. $\mathrm{H}_{2} \mathrm{SO}_{4}$ is an extremely strong oxidizing agent and dehydrates several organic compounds producing carbon in the form of graphite; the same was observed while using conc. sulfuric acid directly as the esterification catalyst. The reaction mixture turned to a solid black mass with a mere yield of $5 \%$. To overcome dehydration and degradation of the reaction mixture, mesoporous solid acid catalysts, silica- $\mathrm{H}_{2} \mathrm{SO}_{4}\left(\mathrm{Si}-\mathrm{H}^{+}\right)$, silicaSA, silica-PTSA, bentonite- $\mathrm{H}_{2} \mathrm{SO}_{4}$, bentonite-SA, and bentonite-PTSA, were designed (Table 1). The \%yield indicated that most of them exhibit significantly improved esterification yields. Interestingly, $\mathrm{Si}-\mathrm{H}^{+}$catalyst, being a superior proton source, affords the highest yield among these catalysts as one site is bonded with $\mathrm{Si}$, while the other end is available to donate proton and carry out reactions under heterogeneous conditions. The silica gel alone did not show any considerable catalytic effects, but $\mathrm{H}_{2} \mathrm{SO}_{4}$ immobilization induced the catalytic activity. However, the mesoporous nature of silica showed enhanced catalytic effects in contrast to bentonite.

After catalyst selection, the concentration was another important factor that was responsible for appreciable yield of the product. For this purpose, different ratios of silica gel and sulfuric acid $(1: 0.25,1: 0.5$, and $1: 0.75)$ were explored 
using 1 mmole of 4-hydroxybenzoic acid and 3 mmole of menthol at $80^{\circ} \mathrm{C}$. The results showed that $1: 0.75$ was the best concentration for esterification on the basis of $\mathrm{H}_{2} \mathrm{SO}_{4}$ properties. In the next step, the same concentration was employed for glycosylation of 2 mmole of $3 \mathrm{a}$ and $1 \mathrm{mmole}$ of $\alpha$-D-glucose at $80^{\circ} \mathrm{C}$ to afford the corresponding glycosides; to our delight, we observed 1:0.75 was equally efficient for glycosylation reducing several steps of protection-deprotection of various functional groups as in traditional glycosylation (Table 2).

To access the catalytic performance of the selected $\mathrm{Si}$ $\mathrm{H}^{+}$catalyst $(1: 0.75)$, different mole $\%$ of the catalyst were utilized at $80^{\circ} \mathrm{C}$. The best amount of the $\mathrm{Si}-\mathrm{H}^{+}$catalyst for 3a was found to be 0.3 mole $\%$ which gave greater yield in short time thus increasing catalyst loading to 0.3 mole $\%$ which actually increased the efficiency of the catalyst due to increased active sites. However, for $\mathbf{5 a}$, any further increase in the amount from 0.1 mole $\%$ resulted in low yield which could be due to dehydration of the glycone (Table 3).

The effect of temperature was studied to locate an effective temperature range for the $\mathrm{Si}-\mathrm{H}^{+}$catalyst $(1: 0.75)$ since its selectivity and catalytic activity are temperaturedependent. The optimum temperature ranges from $70^{\circ} \mathrm{C}$ to $80^{\circ} \mathrm{C}$ with relatively enhanced yield at $80^{\circ} \mathrm{C}$ for most of the esters and glycosides. However, for the compound having steric hindrance, the temperature of $100^{\circ} \mathrm{C}$ was more favorable to carry out the reaction (Table 4 ).

Looking at the effect of reactant ratio showed that the increased amount of menthol in esterification and aglycone in glycosylation enhanced the \%yield of the reaction by driving the equilibrium of the reaction towards the product and limiting the reverse reaction (Table 5). Solvent-free conditions were employed to carry out all the reactions.

Reaction conditions of $3 \mathrm{a}-\mathrm{d}$ and $5 \mathrm{a}-\mathrm{l}$ were optimized with the $\mathrm{Si}-\mathrm{H}^{+}$catalyst $(1: 0.75)$ that demonstrated excellent results according to TLC and FTIR monitoring. Physical data and reaction conditions of $3 a-d$ and $5 a-1$ are summarized in Tables 6 and 7, respectively.

3.2. Catalyst Characterization. The FTIR spectrum of untreated silica gel and $\mathrm{Si}-\mathrm{H}^{+}$catalyst $(1: 0.75)$ was recorded and compared. Catalyst (b) showed small absorption bands at $1400 \mathrm{~cm}^{-1}$ that were assigned to the $\mathrm{S}=\mathrm{O}$ stretching mode. This observation illustrated the immobilization of sulfuric acid on free terminal silanol ( $\mathrm{Si}-\mathrm{OH})$ groups. This further ensured the exchange of $\mathrm{HSO}_{4}^{-}$with $\mathrm{H}$ of the silanol group in silica gel [17]. This stretching was not observed in (a), thus explaining the proposed immobilization of sulfuric acid groups on the free terminal silanol ( $\mathrm{Si}-\mathrm{OH})$ groups in the $\mathrm{Si}-\mathrm{H}^{+}$catalyst (Figure 2). Hence, it was inferred that the irregular tridimensional framework of the porous silica surface allows the insertion of proton due to the presence of siloxane $(\mathrm{Si}-\mathrm{O}-\mathrm{Si})$ and silanol $(\mathrm{Si}-\mathrm{OH})$ groups (Figure 3 ).

A broad band (b) at $3400 \mathrm{~cm}^{-1}$ was observed due to $\mathrm{OH}$ stretching that supported increased hydrogen bonding due to sulfuric acid (Figure 2). The absorption frequency bands observed at 519 and $801 \mathrm{~cm}^{-1}$ were assigned to bending and out-of-plane deformations of $\mathrm{Si}-\mathrm{O}$ bonds which further endorsed successful modifications on the surface of silica, thus proving the formation of catalyst $\mathrm{Si}-\mathrm{H}^{+}$(Table 8 ).

The thermal stability of the $\mathrm{Si}-\mathrm{H}^{+}$catalyst $(1: 0.75)$ and silica was studied via differential scanning calorimetry (DSC) and thermal gravimetric analysis (TGA) from ambient temperature to $800^{\circ} \mathrm{C}$ (Figure 4 ).

The TGA curve of the $\mathrm{Si}-\mathrm{H}^{+}$catalyst (Figure 4(b)) showed two gradual weight losses at $65^{\circ} \mathrm{C}$ and $240^{\circ} \mathrm{C}$ due to the loss of water molecules formed during the catalyst formation and the other from acid. The linear TGA curve after $350^{\circ} \mathrm{C}$ in the spectrum of the catalyst showed greater thermal stability as compared to starting material. The descending TGA thermal curve for silica gel (Figure 4(a)) exhibited abrupt weight loss at $\approx 100^{\circ} \mathrm{C}$ due to the removal of water of hydration. The DSC thermogram of silica gel (Figure 4(a)) showed a large endothermic peak at $100^{\circ} \mathrm{C}$ and a small peak at $770^{\circ} \mathrm{C}$. The sharp endothermic peak of silica gel observed at $100^{\circ} \mathrm{C}$ shifted towards higher temperature at $130^{\circ} \mathrm{C}$ in the $\mathrm{Si}-\mathrm{H}^{+}$catalyst (Figure $4(\mathrm{~b})$ ) that supported the stability of the solid acid catalyst.

The powder XRD patterns of (a) $\mathrm{Si}-\mathrm{H}^{+}$catalyst and (b) silica gel were compared (Figure 5 ). The XRD pattern of both (a) and (b) exhibits one broad $\left(2 \theta=20-30^{\circ}\right)$ and another relatively sharp $\left(2 \theta=03-04^{\circ}\right)$ diffraction peak. The main difference between silica gel and $\mathrm{Si}-\mathrm{H}^{+}$catalyst exists in the emergence of another small diffraction peak in (a) at $2 \theta=11^{\circ}$. The increased intensity of diffraction peaks in the catalyst verified the proposed modification of silica by immobilization of sulfuric acid groups.

The surface morphology of the silica gel and $\mathrm{Si}-\mathrm{H}^{+}$ catalyst was observed by SEM micrographs (Figure 6). These micrographs showed increased surface area and enhanced microporosity of the $\mathrm{Si}-\mathrm{H}^{+}$catalyst in Figure 6(b) as compared to the relatively smooth and regular-shaped silica gel particles (Figure 6(a)).

3.3. Characterization and Thermotropic Behaviour of Aglycones and O-Glycopyranosides. The structures of the nonpolar aglycones (3a-d) and the saccharide derivatives (5a-l) were confirmed by FTIR and ${ }^{1} \mathrm{H}$ NMR spectroscopy. FTIR spectra were scanned from 500 to $4000 \mathrm{~cm}^{-1}$ under dry air at room temperature. A broad band extending from 3300 to $3600 \mathrm{~cm}^{-1}$ was observed corresponding to the stretching vibration of the hydroxyl groups. A distinctive peak above $1700 \mathrm{~cm}^{-1}$ in the FTIR spectrum confirmed the esterification of the menthol hydroxyl group. Absorption peak of $\approx 1100 \mathrm{~cm}^{-1}$ was due to the existence of the ether (C-O-R) linkage between aglycone and saccharide. In ${ }^{1} \mathrm{H}$ NMR spectra, the peaks from 6.72 to $7.88 \mathrm{ppm}$ confirmed the presence of aromatic $\mathrm{C}-\mathrm{H}$ protons in $3 \mathrm{a}-\mathrm{d}$ and $5 \mathrm{a}-\mathrm{l}$. Multiplets were observed at 1.60-1.97 ppm due to the methylene and methyl protons. A singlet at $\approx 9.50 \mathrm{ppm}$ confirmed the presence of the hydroxyl proton.

The TGA and DSC curves for compounds $3 \mathrm{a}-\mathrm{d}$ and $5 \mathrm{a}-\mathrm{l}$ were studied within the varying temperature range of $0-500^{\circ} \mathrm{C}$ with a linear heating rate of $10^{\circ} \mathrm{C} / \mathrm{min}$. The 
TABLE 2: Screening of catalyst concentration.

\begin{tabular}{lccccc}
\hline Entry & Ratio & Ta & & \multicolumn{2}{c}{$5 \mathrm{a}$} \\
& & Time (hr) & Yield (\%) & Time (hr) & 8.5 \\
2 & $1: 0.25$ & 5.5 & 77 & 6.5 & 72 \\
3 & $1: 0.5$ & 2.5 & 80 & 6.25 & 80 \\
\hline
\end{tabular}

TABLE 3: Screening of catalyst loading.

\begin{tabular}{lccccc}
\hline Entry & Amount (mole\%) & 3a & & \multicolumn{2}{c}{$5 \mathrm{a}$} \\
& & Time (hr) & Yield (\%) & Time (hr) & 6.5 \\
1 & 0.1 & 5.5 & 55 & 6.5 & 80 \\
2 & 0.2 & 4.5 & 62 & 6.5 & 67 \\
4 & 0.3 & 2.5 & 71 & 6.25 \\
\hline
\end{tabular}

TABLE 4: Screening of optimum temperature.

\begin{tabular}{lccccc}
\hline Entry & $\begin{array}{c}\text { Temperature } \\
\left({ }^{\circ} \mathrm{C}\right)\end{array}$ & $\begin{array}{c}\text { Time } \\
(\mathrm{hr})\end{array}$ & $\begin{array}{c}\text { Yield } \\
(\%)\end{array}$ & $\begin{array}{c}\text { Time } \\
(\mathrm{hr})\end{array}$ & $\begin{array}{c}\text { Yield } \\
(\%)\end{array}$ \\
\hline 1 & 50 & 6.5 & 54 & 9.5 & 34 \\
2 & 80 & 2.5 & 89 & 7.5 & 81 \\
3 & 100 & 2.25 & 83 & 6.25 & 80 \\
\hline
\end{tabular}

TABLE 5: Screening of reactant ratio.

\begin{tabular}{lccccc}
\hline Entry & $\begin{array}{c}\text { Reactant } \\
\text { ratio }\end{array}$ & $\begin{array}{c}\text { Time } \\
\text { (hr) }\end{array}$ & Yield (\%) & $\begin{array}{c}\text { Time } \\
\text { (hr) }\end{array}$ & Yield (\%) \\
\hline 1 & $1: 1$ & 8.5 & 53 & 9.25 & 48 \\
2 & $1: 2$ & 7.5 & 71 & 7.5 & 54 \\
3 & $1: 3$ & 3.5 & 82 & 6.5 & 80 \\
4 & $1: 4$ & 2.5 & 86 & 6.25 & 83 \\
\hline
\end{tabular}

thermogram of 3a (Figure 7) exhibited a loss of mass at $200-400^{\circ} \mathrm{C}$; this abrupt fall in the TGA curve was attributed to a change of state in the compound, i.e., ordered to disordered state. A glass transition $\left(T_{\mathrm{g}}\right)$ in the DSC thermogram of 3a (Figure 7) was generally observed at $131^{\circ} \mathrm{C}$ lower than the melting point transition $\left(T_{\mathrm{m}}\right)$ at $147^{\circ} \mathrm{C} . T_{\mathrm{g}}$ is the gradual and reversible transition in amorphous and semicrystalline compounds from hard to viscous state as the temperature was increased, while $T_{\mathrm{m}}$ endothermic peak corresponds to the first-order phase transition. The onset of crystallization on the DSC curve of 3 a was observed at $338^{\circ} \mathrm{C}$ with a crystallization exothermic peak at $412^{\circ} \mathrm{C}$ (Figure 7). The onset of crystallization for $3 \mathrm{~b}, 3 \mathrm{c}$, and $3 \mathrm{~d}$ was 308,341 , and 237 , respectively. The inclusion of methoxy groups in $3 \mathrm{~b}$ and $3 \mathrm{c}$ decreases $T_{\mathrm{m}}$, while the increased chain length in $3 c$ causes an increase in $T_{\mathrm{m}}$ as compared with $3 \mathrm{a}$. Phase transition temperatures observed for $3 \mathrm{a}-\mathrm{d}$ compounds using DSC are stated in Table 9.

TGA curves of $5 \mathrm{a}$ showed several gradual weight loss events at $112-137^{\circ} \mathrm{C}, 150-187^{\circ} \mathrm{C}$, and $200-250^{\circ} \mathrm{C}$. The DSC curve of $5 \mathrm{a}$ showed that it melted through the mesophases with two varying melting point transitions (Figure 8).

First, the hydrocarbon part melted at low temperature $\left(T_{\mathrm{m}}^{1}\right)$ at $75^{\circ} \mathrm{C}$ resulting in the loss of the three-dimensional framework of soft hydrocarbon crystals. However, the hard crystals that correspond to the carbohydrate part melted at a higher temperature $\left(T_{\mathrm{m}}^{2}\right)$ at $134^{\circ} \mathrm{C}$ and formed isotropic liquid. In between the two melting points, the mesophases appeared to be stable at $117^{\circ} \mathrm{C}$ in $5 \mathrm{a}$. An exothermic peak at a lower temperature of $28^{\circ} \mathrm{C}$ is also related to the carbohydrate liquid crystals. With an onset of crystallization at $200^{\circ} \mathrm{C}$, two exothermic crystalline peaks are observed at 219 and $283^{\circ} \mathrm{C}$. The two melting point transitions in glycosides 5a-l supported the thermotropic mesophase behaviour including various crystal-to-crystal, crystal-to-mesophase and mesophase-to-mesophase transitions [5]. Phase transition temperatures supporting the mesogenic character of synthesized carbohydrate derivatives $(5 \mathrm{a}-\mathrm{l})$ are stated in Table 10.

The mesogenic textures of the amphiphilic glycopyranosides $(5 \mathrm{a}-\mathrm{l})$ were observed by polarized optical microscopy under crossed polarizers. All the glycopyranosides showed the formation of thermotropic mesophases during heating and cooling of the pure samples. For compound $5 \mathrm{a}$, the mesophase was observed between 80 and $130^{\circ} \mathrm{C}$ with a fan/focal-conical texture (Figure 9(a)) that is common for the smectic A (SmA) phases. In Figure 9(b), typical focal conics of the SmA phase were observed for compound $5 \mathrm{~b}$ between 65 and $125^{\circ} \mathrm{C}$, while Figure 9(c) shows fan-shaped focal-conical SmA phases of compound $5 \mathrm{c}$ between 70 and $150^{\circ} \mathrm{C}$. Compound $5 \mathrm{e}$ showed $\mathrm{SmA}$ phase droplets (Figure $10(\mathrm{a})$ ) at $110^{\circ} \mathrm{C}$ with phase transition temperature between 60 and $125^{\circ} \mathrm{C}$. SmA phase of compound 5h with focal-conical defects (Figure 10(b)) was visible between 80 and $160^{\circ} \mathrm{C}$. SmA phase focal-conical texture was also observed for compound $5 \mathrm{k}$ (Figure $10(\mathrm{c})$ ) at $90^{\circ} \mathrm{C}$. In addition to crystal-to-crystal transitions at lower temperature and existence of double melting points, the formation of smectic thermotropic phases supported the liquid crystalline nature of synthesized glycopyranosides. 
TABLE 6: Summary of physical data and reaction conditions of nonpolar aglycones (3a-3d).

\begin{tabular}{|c|c|c|c|c|c|c|}
\hline Product code & Product structure & Molecular formula & Time $(\mathrm{hr})$ & Temperature, $T\left({ }^{\circ} \mathrm{C}\right)$ & $\mathrm{R}_{f}$ value & Yield (\%) \\
\hline $3 a$ & & $\mathrm{C}_{17} \mathrm{H}_{24} \mathrm{O}_{3}$ & 2.5 & 80 & 0.75 & 89 \\
\hline $3 b$ & & $\mathrm{C}_{18} \mathrm{H}_{26} \mathrm{O}_{4}$ & 3 & 80 & 0.66 & 93 \\
\hline $3 c$ & & $\mathrm{C}_{19} \mathrm{H}_{28} \mathrm{O}_{5}$ & 4.5 & 100 & 0.57 & 82 \\
\hline $3 d$ & & $\mathrm{C}_{20} \mathrm{H}_{28} \mathrm{O}_{4}$ & 1.5 & 70 & 0.60 & 96 \\
\hline
\end{tabular}

TABLE 7: Summary of physical data and reaction conditions of $5 \mathrm{a}-51$.

\begin{tabular}{|c|c|c|c|c|c|c|}
\hline Product code & Compound structure & Molecular formula & Time (hr) & Temperature, $T\left({ }^{\circ} \mathrm{C}\right)$ & $\mathrm{R}_{f}$ value & Yield (\%) \\
\hline $5 a$ & & $\mathrm{C}_{23} \mathrm{H}_{34} \mathrm{O}_{8}$ & 6.5 & 80 & 0.77 & 80 \\
\hline $5 b$ & & $\mathrm{C}_{22} \mathrm{H}_{32} \mathrm{O}_{7}$ & 6.25 & 80 & 0.68 & 85 \\
\hline $5 c$ & & $\mathrm{C}_{29} \mathrm{H}_{44} \mathrm{O}_{13}$ & 6.5 & 80 & 0.52 & 84 \\
\hline $5 \mathrm{~d}$ & & $\mathrm{C}_{24} \mathrm{H}_{36} \mathrm{O}_{9}$ & 8.5 & 90 & 0.86 & 82 \\
\hline $5 e$ & & $\mathrm{C}_{23} \mathrm{H}_{34} \mathrm{O}_{8}$ & 8.25 & 90 & 0.73 & 71 \\
\hline
\end{tabular}


TABle 7: Continued.

\begin{tabular}{|c|c|c|c|c|c|c|}
\hline Product code & Compound structure & Molecular formula & Time (hr) & Temperature, $T\left({ }^{\circ} \mathrm{C}\right)$ & $\mathrm{R}_{f}$ value & Yield (\%) \\
\hline $5 f$ & & $\mathrm{C}_{30} \mathrm{H}_{46} \mathrm{O}_{14}$ & 9.25 & 90 & 0.65 & 80 \\
\hline $5 \mathrm{~g}$ & & $\mathrm{C}_{25} \mathrm{H}_{38} \mathrm{O}_{10}$ & 9.5 & 100 & 0.89 & 74 \\
\hline $5 \mathrm{~h}$ & & $\mathrm{C}_{24} \mathrm{H}_{36} \mathrm{O}_{9}$ & 9.25 & 100 & 0.81 & 69 \\
\hline $5 i$ & & $\mathrm{C}_{31} \mathrm{H}_{48} \mathrm{O}_{15}$ & 9.5 & 100 & 0.73 & 68 \\
\hline $5 j$ & & $\mathrm{C}_{26} \mathrm{H}_{38} \mathrm{O}_{9}$ & 6.5 & 75 & 0.57 & 83 \\
\hline $5 \mathrm{k}$ & & $\mathrm{C}_{25} \mathrm{H}_{36} \mathrm{O}_{8}$ & 6.5 & 80 & 0.61 & 76 \\
\hline 51 & & $\mathrm{C}_{32} \mathrm{H}_{48} \mathrm{O}_{14}$ & 7.25 & 80 & 0.73 & 65 \\
\hline
\end{tabular}

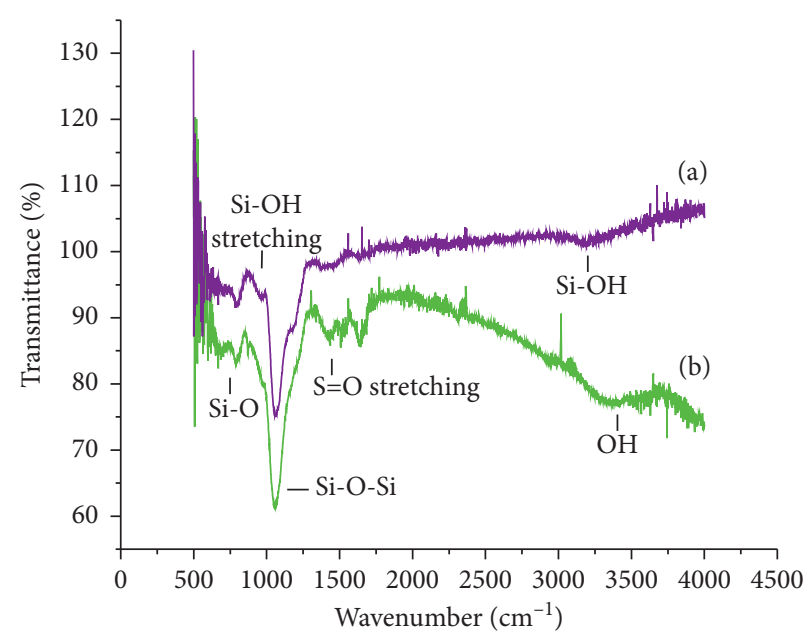

FIgURe 2: FTIR spectra of (a) silica gel and (b) Si- $\mathrm{H}^{+}$catalyst. 


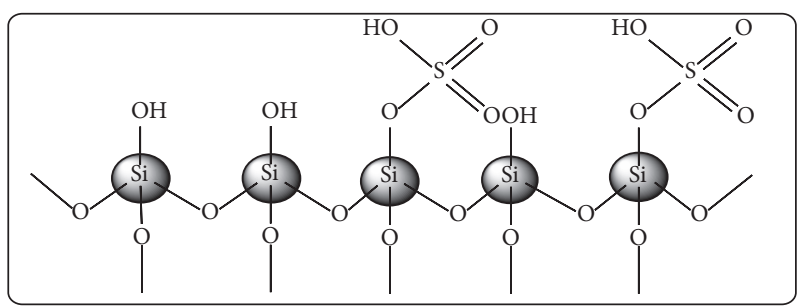

Figure 3: Proposed structure of the $\mathrm{Si}-\mathrm{H}^{+}$catalyst.

TABLE 8: Characteristic FTIR absorption bands of silica-supported acid catalysts.

\begin{tabular}{lccccccc}
\hline \multirow{2}{*}{ Entry } & \multirow{2}{*}{ Catalysts } & Si-O & Si-O bending & Si-OH stretching & Si-O-Si & Si-OH & S=O \\
\hline 1 & & $\mathrm{SiO}_{2}$ & 519 & 801 & 967 & 1057 & 3250 \\
2 & $\mathrm{SiO}_{2}-\mathrm{H}_{2} \mathrm{SO}_{4}$ & 597 & 811 & 871 & 1054 & 3400 \\
\hline
\end{tabular}

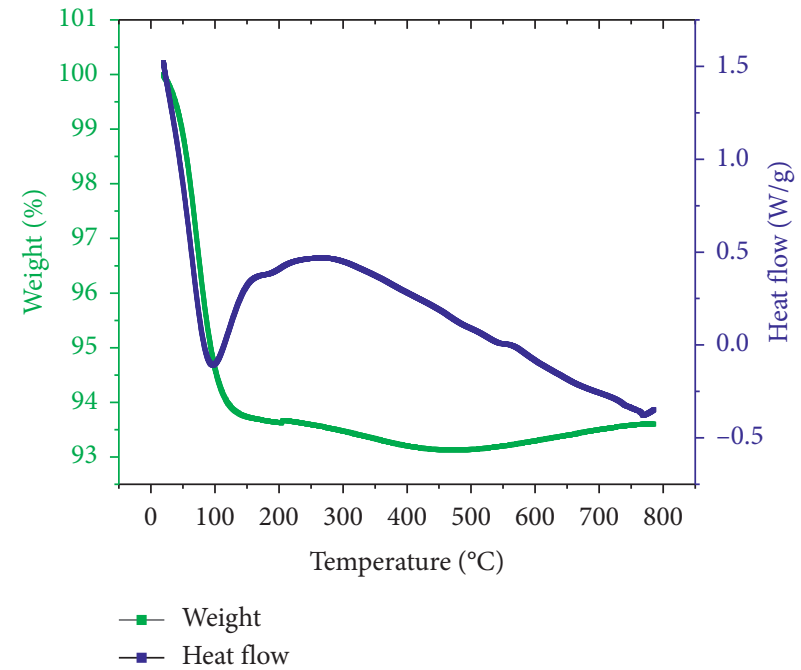

(a)

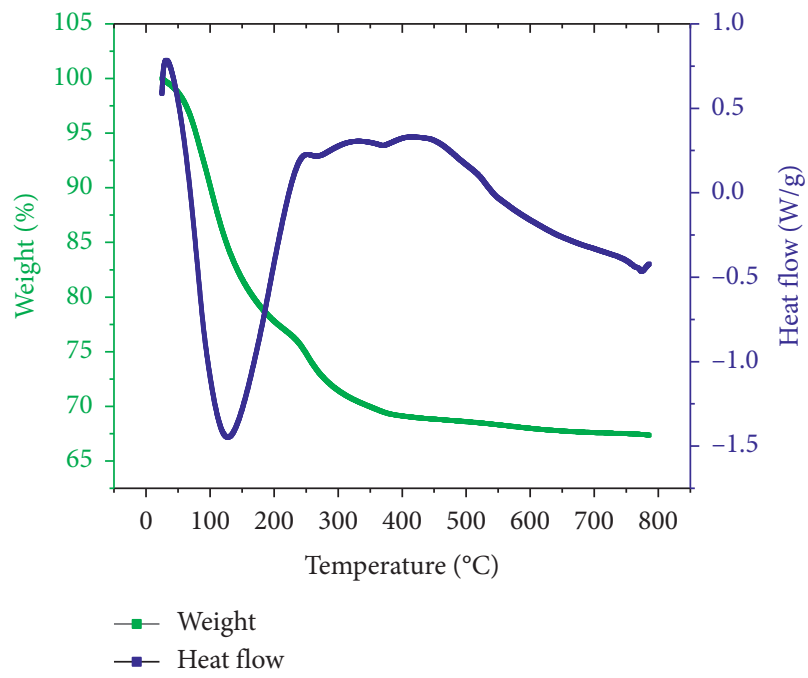

(b)

FIgURE 4: DSC and TGA profiles of (a) silica gel and (b) Si- $\mathrm{H}^{+}$catalyst.

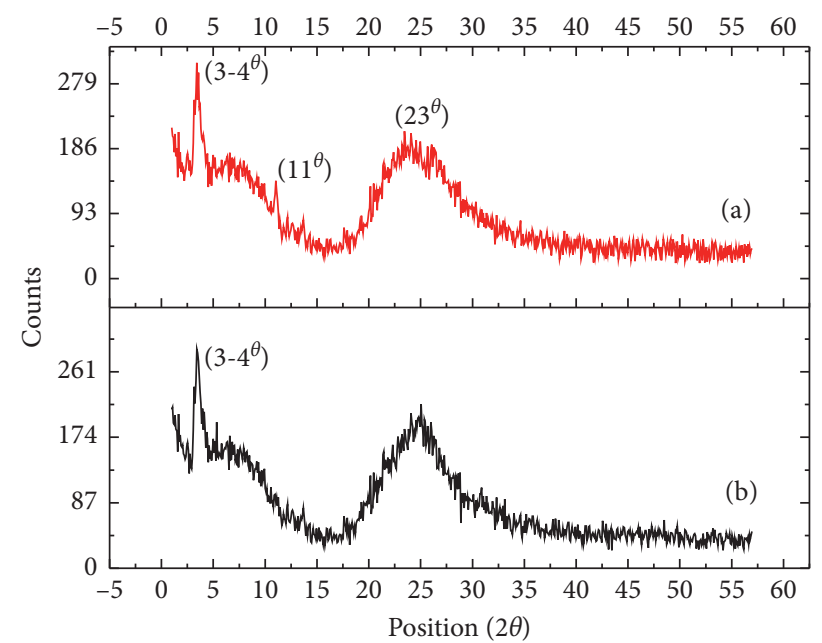

Figure 5: XRD patterns of (a) Si-H $\mathrm{H}^{+}$catalyst and (b) silica gel. 


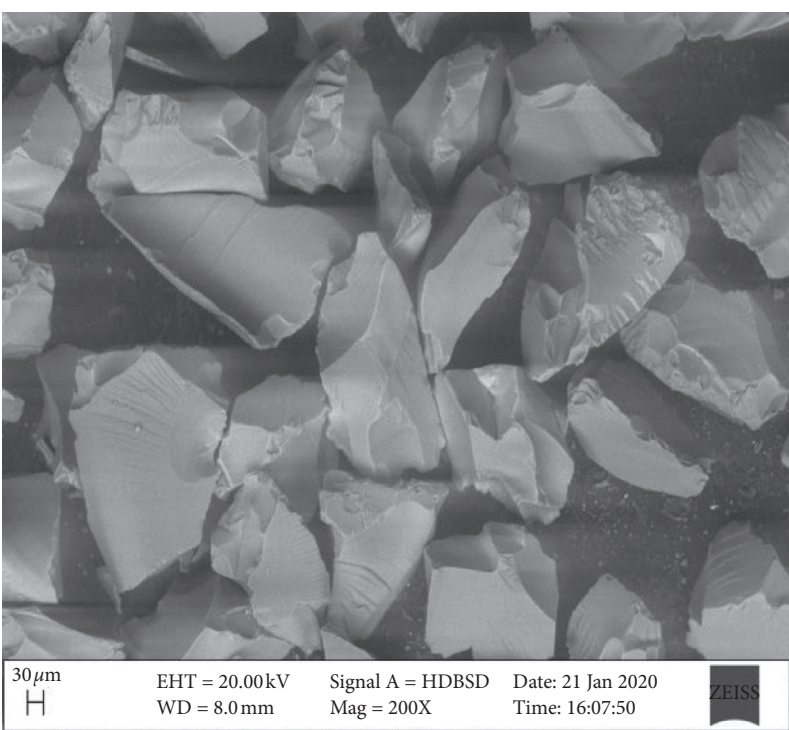

(a)

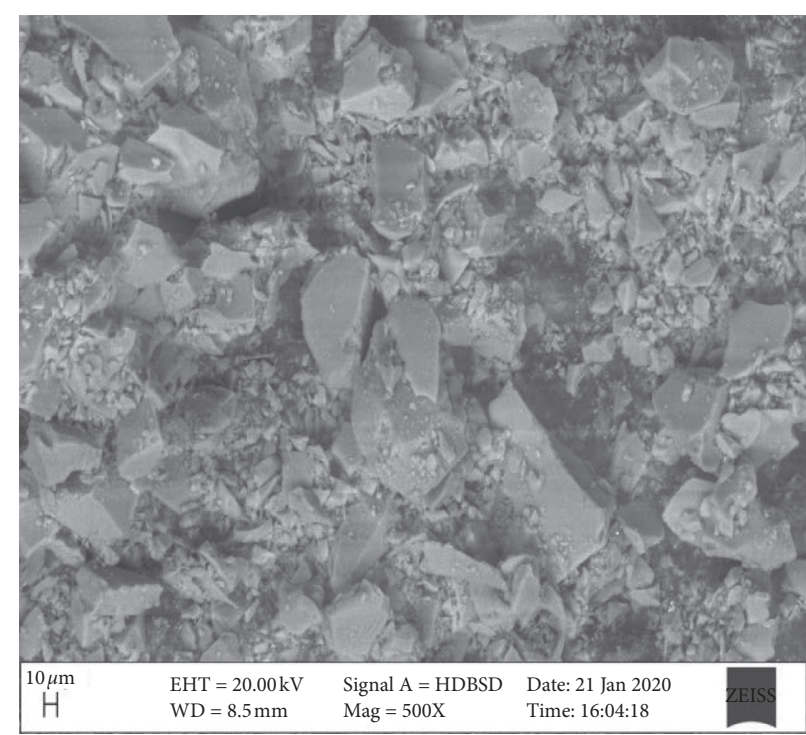

(b)

FIgURE 6: SEM photograph of (a) silica gel and (b) $\mathrm{Si}-\mathrm{H}^{+}$catalyst.

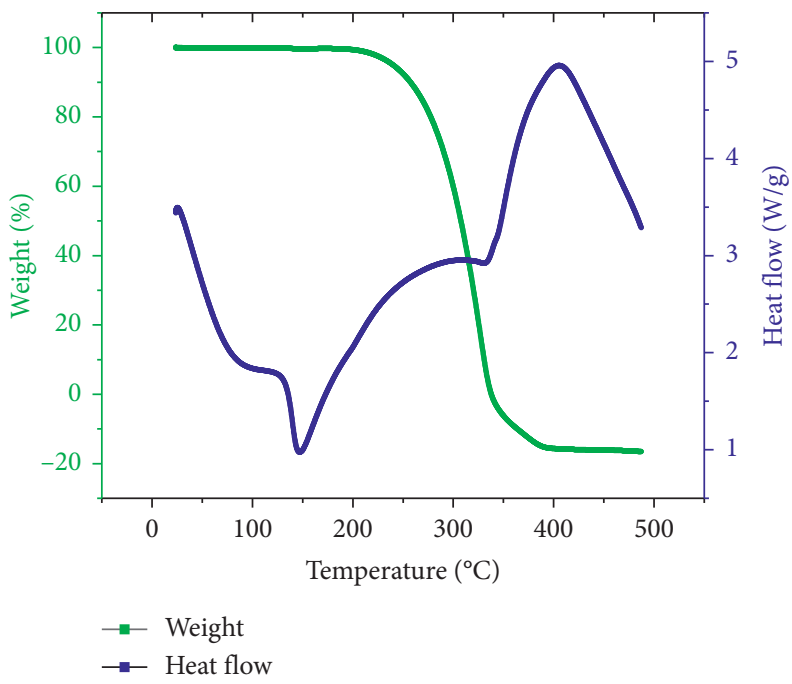

Figure 7: DSC and TGA thermogram of $3 \mathrm{a}$.

TABLE 9: DSC transition temperatures of phenolic ester aglycones.

\begin{tabular}{lcccc}
\hline $\begin{array}{l}\text { Product } \\
\text { code }\end{array}$ & $\begin{array}{c}\text { Melting point, } T_{\mathrm{m}} \\
\left({ }^{\circ} \mathrm{C}\right)\end{array}$ & $\begin{array}{c}\text { Crystallization point, } T_{\mathrm{Cr}} \\
\left({ }^{\circ} \mathrm{C}\right)\end{array}$ & $\begin{array}{c}\text { Glass transition temperature, } T_{\mathrm{g}} \\
\left({ }^{\circ} \mathrm{C}\right)\end{array}$ & $\begin{array}{c}\text { Temperature range of TGA mass loss } \\
\left({ }^{\circ} \mathrm{C}\right)\end{array}$ \\
\hline $3 \mathrm{a}$ & 147 & 412 & 31 & $200-400$ \\
$3 \mathrm{~b}$ & 131 & 387 & 112 & $200-325$ \\
$3 \mathrm{c}$ & 108 & 358 & - & $200-350$ \\
$3 \mathrm{~d}$ & 256 & 387 & 218 & $200-300$ \\
\hline
\end{tabular}




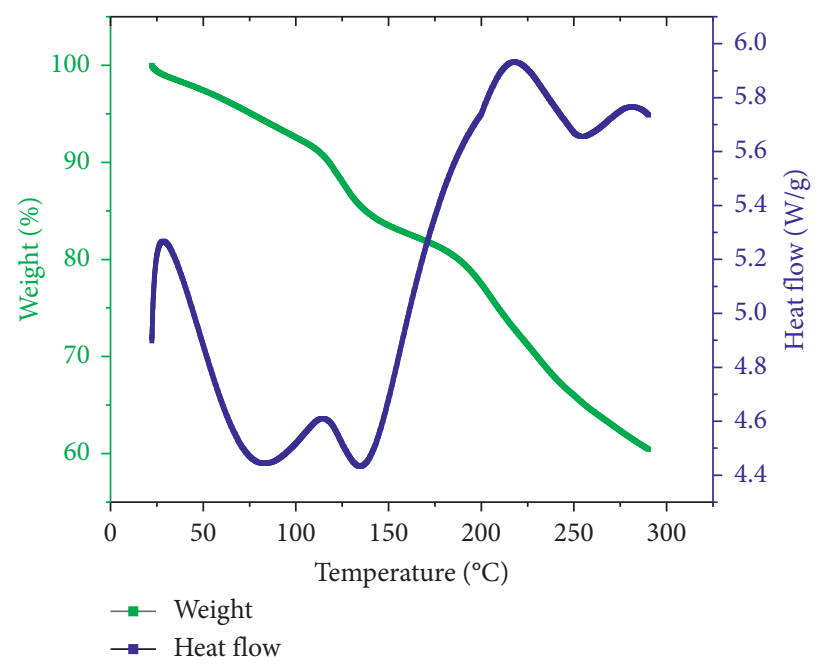

Figure 8: DSC and TGA thermogram of 5 a.

TABLE 10: DSC transition temperatures of O-glycopyranosides.

\begin{tabular}{lcccc}
\hline Product code & $1^{\text {st }}$ melting point, $T_{\mathrm{m}}^{1}\left({ }^{\circ} \mathrm{C}\right)$ & $2^{\text {nd }}$ melting point, $T_{\mathrm{m}}^{2}\left({ }^{\circ} \mathrm{C}\right)$ & Crystallization point, $T_{\mathrm{Cr}}\left({ }^{\circ} \mathrm{C}\right)$ & Glass transition temperature, $T_{\mathrm{g}}\left({ }^{\circ} \mathrm{C}\right)$ \\
\hline $5 \mathrm{a}$ & 75 & 134 & $28,117,219,283$ & - \\
$5 \mathrm{~b}$ & 67 & 133 & 31,225 & 100 \\
$5 \mathrm{c}$ & 71 & 146 & 17,250 & 106 \\
$5 \mathrm{~d}$ & 87 & 287 & $183,221,254$ & - \\
$5 \mathrm{e}$ & 63 & 125 & 112,212 & - \\
$5 \mathrm{f}$ & 275 & $33,250,334,434$ & 217 \\
$5 \mathrm{~g}$ & 125 & 102 & 33,390 & - \\
$5 \mathrm{~h}$ & 95 & 167 & $145,320,375$ & 115 \\
$5 \mathrm{i}$ & 85 & 325 & $25,265,385$ & 130 \\
$5 \mathrm{j}$ & 130 & 150 & $30,265,410$ & - \\
$5 \mathrm{k}$ & 87 & 110 & $25,220,360$ & 110 \\
$5 \mathrm{l}$ & 65 & 166 & 35,275 & \\
\hline
\end{tabular}

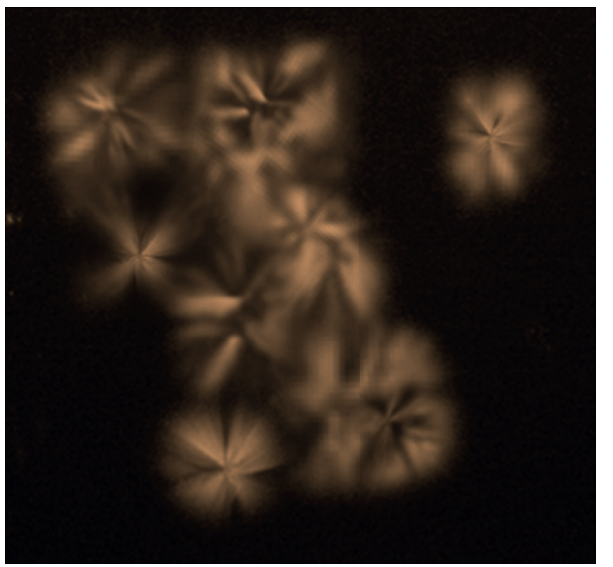

(a)

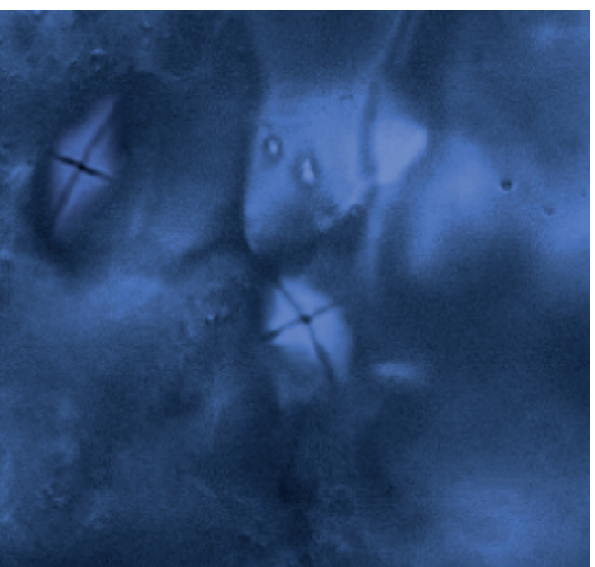

(b)

FIgURE 9: Continued. 


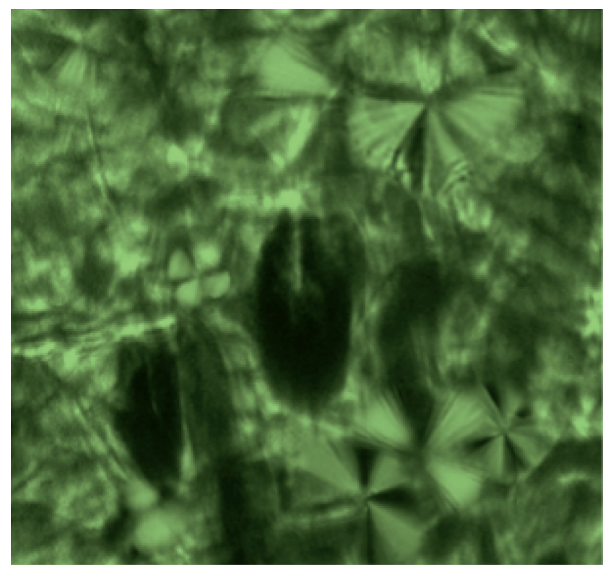

(c)

Figure 9: Optical photomicrographs of (a) compound 5a $\left(80^{\circ} \mathrm{C}, 40 \mathrm{x}\right)$, (b) compound $5 \mathrm{~b}\left(89^{\circ} \mathrm{C}, 10 \mathrm{x}\right)$, and $(\mathrm{c})$ compound $5 \mathrm{c}\left(75^{\circ} \mathrm{C}, 10 \mathrm{x}\right)$ showing mesophase textures.

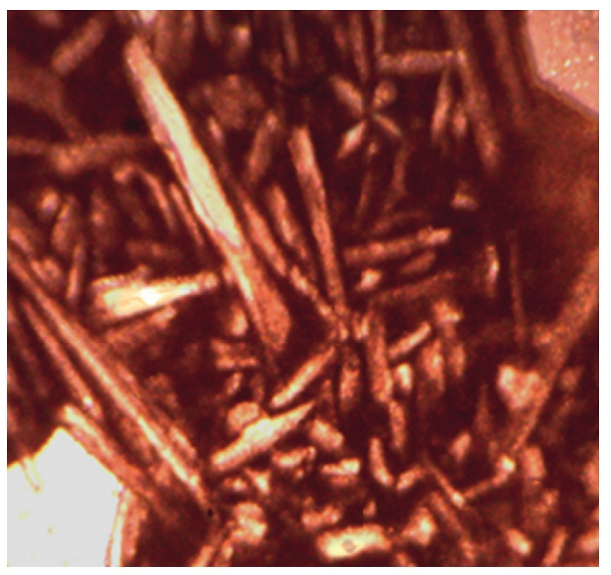

(a)

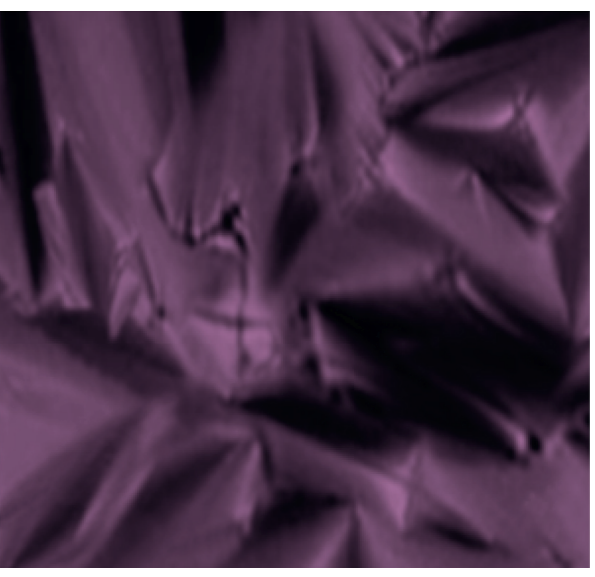

(b)

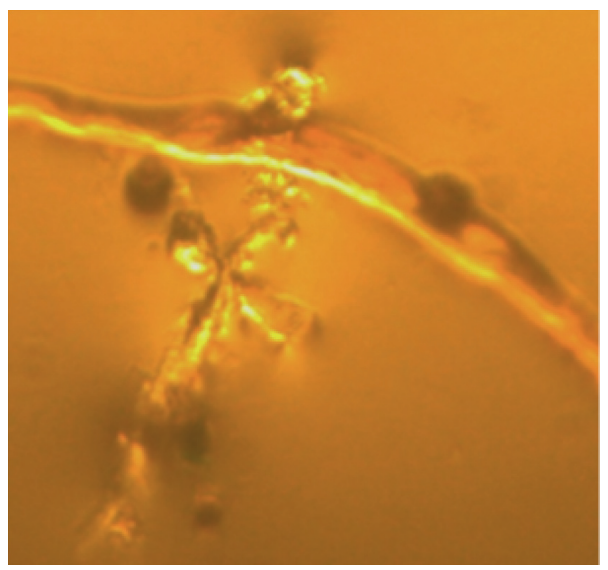

(c)

Figure 10: Optical photomicrographs of (a) compound $5 \mathrm{e}\left(110^{\circ} \mathrm{C}, 10 \mathrm{x}\right),(\mathrm{b})$ compound $5 \mathrm{~h}\left(85^{\circ} \mathrm{C}, 10 \mathrm{x}\right)$, and $(\mathrm{c})$ compound $5 \mathrm{k}\left(90^{\circ} \mathrm{C}, 10 \mathrm{x}\right)$ showing mesophase textures. 


\section{Conclusion}

In conclusion, a study of the mesogenic behaviour of O-glycopyranosides (5a-1), derived from various monosaccharides and disaccharides, has shown that all the compounds have double melting point endothermic and crystalline exothermic peaks in DSC thermograms. The presence of the phenyl ring stabilizes the mesophases of the compounds and results in the formation of thermotropic phases. Optical textures of compounds $5 \mathrm{a}-\mathrm{c}, 5 \mathrm{e}, 5 \mathrm{~h}$, and $5 \mathrm{k}$ displayed smectic A phases. These results for improved potentials of the solid-supported $\mathrm{Si}-\mathrm{H}^{+}$catalyst for glycosylation in solvent-free conditions will greatly contribute to the practical synthesis of glycosides in an environmentally acceptable manner and their use as thermotropic liquid crystals in various biomedical and material applications.

\section{Data Availability}

The data used to support the findings of this study are included within the article.

\section{Conflicts of Interest}

The authors declare that they have no conflicts of interest.

\section{Acknowledgments}

The authors thank the Higher Education Commission, Pakistan, for providing funds under the HEC Indigenous PhD Scholarship to Saliha Nazir and ICCBS, H.E.J. Research Institute of Chemistry, University of Karachi, for providing proton NMR faciltity.

\section{References}

[1] E. Smiths, J. B. F. N. Engberts, R. M. Kellogg, and H. A. VanDoren, "Reliable method for the synthesis of aryl$\beta$-D-glucopyranosides, using boron trifluoride-diethyl ether as catalyst," Journal of the Chemical Society, Perkins Transactions, vol. 1, no. 24, pp. 2873-2877, 1996.

[2] E. Smiths, J. B. F. N. Engberts, R. M. Kellogg, and H. A. VanDoren, "Thermotropic and lyotropic liquid crystalline behavior of 4-alkoxyphenyl $\beta$-D-glucopyranosides," Liquid Crystals, vol. 23, no. 4, pp. 481-488, 1997.

[3] G. A. Jeffrey and L. M. Wingert, "Carbohydrate liquid crystals,” Liquid Crystals, vol. 12, no. 2, pp. 179-202, 1992.

[4] V. Vill and R. Hashim, "Carbohydrate liquid crystals: structure-property relationship of thermotropic and lyotropic glycolipids," Current Opinion in Colloid and Interface Science, vol. 7, no. 5-6, pp. 395-409, 2002.

[5] A. R. N. M. Abeyrathne, A. D. L. C. Perera, and D. N. Karunaratne, "Liquid crystal behaviour of three novel glycosides," Journal of the National Science Foundation of Sri Lanka, vol. 40, no. 2, pp. 115-121, 2012.

[6] M. Veber, E. Cheylan, S. Czernecki, and J. Xie, "Synthesis and mesomorphic properties of some aryl $\beta$-D-C-glycosides," Liquid Crystals, vol. 21, no. 2, pp. 197-201, 1996.

[7] V. Adasch, B. Hoffmann, W. Milius, G. Platz, and G. Voss, "Preparation of alkyl $\alpha$ - and $\beta$-d-Glucopyranosides, thermotropic properties and X-ray analysis," Carbohydrate Research, vol. 314, no. 3-4, pp. 177-187, 1998.
[8] R. Beaulieu, S. Gottis, C. Meyer et al., "Cholesteryl and diosgenyl glycosteroids: synthesis and characterization of new smectic liquid crystals," Carbohydrate Research, vol. 404, pp. 70-78, 2015.

[9] M. K. Singh, N. Jayaraman, D. S. S. Rao, and S. K. Prasad, "Role of hydroxyl group on the mesomorphism of alkyl glycosides: synthesis and thermal behavior of alkyl 6-deoxy$\beta$-d-glucopyranosides," Chemistry and Physics of Lipids, vol. 163, no. 6, pp. 580-585, 2010.

[10] M. J. Clemente, J. Fitremann, M. Mauzac, J. L. Serrano, and L. Oriol, "Synthesis and characterization of maltose-based amphiphiles as supramolecular hydrogelators," Langmuir, vol. 27 , no. 24 , pp. 15236-15247, 2011.

[11] M. K. Singh, D. Swain, T. N. Guru Row, and N. Jayaraman, "Crystal structures and thermal analyses of alkyl 2-deoxy- $\alpha$-darabino-hexopyranosides," Carbohydrate Research, vol. 344, no. 15, pp. 1993-1998, 2009.

[12] T. Tsuruta, T. Koyama, M. Yasutake, K. Hatano, and K. Matsuoka, "Synthesis of chiral dopants based on carbohydrates," Carbohydrate Research, vol. 393, pp. 15-22, 2014.

[13] R. Hashim, H. Hassan, A. S. Hamzah, V. Vill, and M. Wulf, "Synthesis of branched-chain alkyl glucosides and their liquid crystal behaviour," Electronic-Liquid Crystal Communications, pp. 1-17, 2003.

[14] A. Y. El-Naggar, "Thermal analysis of the modified and unmodified silica gels to estimate their applicability as stationary phase in gas chromatography," Journal of Emerging Trends in Engineering and Applied Sciences, vol. 4, no. 1, pp. 144-148, 2013.

[15] N. Z. B. M. Rodzi, T. Heidelberg, R. Hashim, A. Sugimura, and H. Minamikawa, "Synthesis and liquid crystals properties of $\alpha$-methylated galactosides," Physics Procedia, vol. 14, pp. 91-95, 2011.

[16] N. J. Brooks, H. A. A. Hamid, R. Hashim et al., "Thermotropic and lyotropic liquid crystalline phases of guerbet branchedchain -D-Glucosides," Liquid Crystals, vol. 38, no. 11-12, pp. 1725-1734, 2011.

[17] N. Laurent, D. Lafont, F. Dumoulin et al., "Synthesis of amphiphilic phenylazophenyl glycosides and a study of their liquid crystal properties," Journal of the American Chemical Society, vol. 125, no. 50, pp. 15499-15506, 2003.

[18] L. Yang, Y. Dong, X. Hu, and A. Liu, "Synthesis and liquid crystallinity of dendronized carbohydrate liquid crystal," Carbohydrate Research, vol. 347, no. 1, pp. 40-46, 2012.

[19] S. Narayanaperumal, R. C. D. Silva, J. L. Monteiro, A. G. Paixão, and M. W. Paixao, "Iron(III) chloride catalyzed glycosylation of peracylated sugars with allyl/alkynyl alcohols," Journal of the Brazilian Chemical Society, vol. 23, no. 11, pp. 1982-1988, 2012.

[20] N. Lubin-Germain, J.-P. Baltaze, A. Coste et al., "DirectC-glycosylation by indium-mediated alkynylation on sugar anomeric position," Organic Letters, vol. 10, no. 5, pp. 725-728, 2008.

[21] T. H. T. Vu, M. H. Nguyen, and M. D. Nguyen, "Synthesis of acidic heterogeneous catalysts with high stability based on graphene oxide/activated carbon composites for the esterification of lactic acid," Journal of Chemistry, vol. 2019, Article ID 7815697, 7 pages, 2019.

[22] P. Gupta and S. Paul, "Solid acids: green alternatives for acid catalysis," Catalysis Today, vol. 236, pp. 153-170, 2014.

[23] J. H. Clark, S. Elings, and K. Wilson, "Catalysis for green chemistry: ultrahigh loaded mesoporous solid acids," Comptes Rendus de l'Académie des Sciences-Series IIC-Chemistry, vol. 3, no. 6, pp. 399-404, 2000. 